\title{
FUGLEDE'S SPECTRAL SET CONJECTURE FOR CONVEX POLYTOPES
}

\author{
RACHEL GREENFELD AND NIR LEV
}

\begin{abstract}
Let $\Omega$ be a convex polytope in $\mathbb{R}^{d}$. We say that $\Omega$ is spectral if the space $L^{2}(\Omega)$ admits an orthogonal basis consisting of exponential functions. There is a conjecture, which goes back to Fuglede (1974), that $\Omega$ is spectral if and only if it can tile the space by translations. It is known that if $\Omega$ tiles then it is spectral, but the converse was proved only in dimension $d=2$, by Iosevich, Katz and Tao.

By a result due to Kolountzakis, if a convex polytope $\Omega \subset \mathbb{R}^{d}$ is spectral, then it must be centrally symmetric. We prove that also all the facets of $\Omega$ are centrally symmetric. These conditions are necessary for $\Omega$ to tile by translations.

We also develop an approach which allows us to prove that in dimension $d=3$, any spectral convex polytope $\Omega$ indeed tiles by translations. Thus we obtain that Fuglede's conjecture is true for convex polytopes in $\mathbb{R}^{3}$.
\end{abstract}

\section{INTRODUCTION}

1.1. Let $\Omega \subset \mathbb{R}^{d}$ be a bounded, measurable set of positive Lebesgue measure. A countable set $\Lambda \subset \mathbb{R}^{d}$ is called a spectrum for $\Omega$ if the system of exponential functions

$$
E(\Lambda)=\left\{e_{\lambda}\right\}_{\lambda \in \Lambda}, \quad e_{\lambda}(x)=e^{2 \pi i\langle\lambda, x\rangle},
$$

constitutes an orthogonal basis in $L^{2}(\Omega)$, that is, the system is orthogonal and complete in the space. A set $\Omega$ which admits a spectrum $\Lambda$ is called a spectral set.

The classical example of such a situation is when $\Omega$ is the unit cube in $\mathbb{R}^{d}$, and $\Lambda$ is the integer lattice $\mathbb{Z}^{d}$. Which other sets $\Omega$ are spectral? The study of this problem was initiated by Fuglede in 1974 [Fug74]. For example, in that paper it was shown that a triangle and a disk in the plane are not spectral sets.

The set $\Omega$ is said to tile the space by translations along a countable set $\Lambda \subset \mathbb{R}^{d}$ if the family of sets $\Omega+\lambda(\lambda \in \Lambda)$ constitutes a partition of $\mathbb{R}^{d}$ up to measure zero. In this case we will say that $\Omega+\Lambda$ is a tiling. Fuglede observed in Fug74 the following connection between the concepts of spectrality and tiling:

Let $\Lambda$ be a lattice. If $\Omega+\Lambda$ is a tiling, then the dual lattice $\Lambda^{*}$ is a spectrum for $\Omega$, and also the converse is true.

Here, by a lattice we mean the image of $\mathbb{Z}^{d}$ under some invertible linear transformation, and the dual lattice is the set of all vectors $\lambda^{*}$ such that $\left\langle\lambda, \lambda^{*}\right\rangle \in \mathbb{Z}, \lambda \in \Lambda$.

Fuglede conjectured that the spectral sets could be characterized in geometric terms using the concept of tiling, in the following way: the set $\Omega$ is spectral if and only if it can tile the space by translations. This conjecture inspired extensive research over the

Date: May 29, 2017.

2010 Mathematics Subject Classification. 42B10, 52C22.

Key words and phrases. Fuglede's conjecture, spectral set, tiling, convex polytope.

Research supported by ISF grant No. 225/13 and ERC Starting Grant No. 713927. 
years, and a number of interesting results supporting the conjecture had been obtained. See, for example, the survey given in [Kol04, Section 3].

On the other hand, it turned out that there also exist counter-examples to Fuglede's conjecture. In [Tao04], Tao constructed in dimensions 5 and higher an example of a set $\Omega$ which is spectral, but cannot tile by translations. Subsequently, also examples of nonspectral sets which can tile by translations were found, and eventually the dimension in these examples was reduced up to $d \geqslant 3$ (see [KM10, Section 4] and the references mentioned there). In all these examples the set $\Omega$ is the union of a finite number of unit cubes centered at points of the integer lattice $\mathbb{Z}^{d}$.

1.2. It is nevertheless believed that Fuglede's conjecture should be true if the set $\Omega$ is assumed to be convex. There is a well-known characterization due to Venkov Ven54, that was rediscovered by McMullen [McM80, McM81], of the convex bodies (compact convex sets with non-empty interior) which can tile the space by translations:

Let $\Omega$ be a convex body in $\mathbb{R}^{d}$. Then $\Omega$ tiles by translations if and only if the following four conditions are satisfied:

(i) $\Omega$ is a polytope;

(ii) $\Omega$ is centrally symmetric;

(iii) all the facets of $\Omega$ are centrally symmetric;

(iv) each "belt" of $\Omega$ consists of exactly 4 or 6 facets.

By a belt of a convex polytope $\Omega \subset \mathbb{R}^{d}$ with centrally symmetric facets one means the collection of its facets which contain a translate of a given subfacet (that is, a $(d-2)$-dimensional face) of $\Omega$.

It was also proved in [Ven54, McM80] that if a convex polytope $\Omega$ can tile by translations, then it admits a face-to-face tiling by translates along a certain lattice. Hence, combined with Fuglede's theorem above this yields the following result:

Let $\Omega \subset \mathbb{R}^{d}$ be a convex body. If $\Omega$ tiles by translations, then $\Omega$ is spectral.

The converse to this result, however, is known only in dimension $d=2$. It is due to Iosevich, Katz and Tao [IKT03, who showed that a spectral convex body in $\mathbb{R}^{2}$ must be either a parallelogram or a centrally symmetric hexagon, and hence it tiles by translations.

The situation in dimensions $d \geqslant 3$ is much less understood. It is known that the ball is not a spectral set [KP99, Fug01, as well as any convex body with a smooth boundary [IKT01]. We established in [GL16] that if $\Omega$ is a cylindric convex body whose base has a smooth boundary, then it can neither be spectral.

Kolountzakis [Kol00] proved the following result:

Let $\Omega$ be a convex body in $\mathbb{R}^{d}$. If $\Omega$ is spectral, then it must be centrally symmetric.

1.3. In this paper we will focus on the case when $\Omega$ is a convex polytope. Our first result shows that in this case, not only the central symmetry of $\Omega$, but also the central symmetry of all the facets of $\Omega$, is a necessary condition for spectrality:

Theorem 1.1. Let $\Omega$ be a convex polytope in $\mathbb{R}^{d}$. If $\Omega$ is a spectral set, then all the facets of $\Omega$ must be centrally symmetric. 
Our proof of this result is inspired by the paper [KP02].

Together with the result from [Kol00] we thus obtain that a spectral convex polytope $\Omega \subset \mathbb{R}^{d}$ must satisfy the conditions (ii) and (iii) in the Venkov-McMullen theorem above. So this supports the conjecture that any such $\Omega$ can tile by translations.

Our next theorem, which is the main result of this paper, confirms that this is indeed the case in dimension $d=3$ :

Theorem 1.2. Let $\Omega$ be a convex polytope in $\mathbb{R}^{3}$. If $\Omega$ is a spectral set, then it can tile by translations.

Combined with the above mentioned results we thus obtain that Fuglede's conjecture is true for convex polytopes $\Omega \subset \mathbb{R}^{3}$.

1.4. In two dimensions, the convex polygons which can tile by translations are precisely the parallelograms and the centrally symmetric hexagons. The three-dimensional convex polytopes which can tile by translations were classified in 1885 by Fedorov [Fed85. into five distinct combinatorial types: the parallelepiped, the hexagonal prism, the rhombic dodecahedron, the elongated dodecahedron and the truncated octahedron (see, for example, Gru07, Figure 32.4] for a graphical illustration of these types). Thus, for a convex polytope $\Omega \subset \mathbb{R}^{3}$ to tile by translations it is necessary and sufficient that it belongs to one of these five types, and that $\Omega$, as well as all its facets, are centrally symmetric. A detailed exposition of this result can be found in [Ale05, Section 8.1].

Theorem 1.2 therefore yields that these conditions are also necessary and sufficient for a convex polytope $\Omega \subset \mathbb{R}^{3}$ to be spectral.

(The requirement that $\Omega$ is centrally symmetric is in fact redundant in this characterization: it is known Ale33. that if all the facets of a convex polytope $\Omega \subset \mathbb{R}^{d}, d \geqslant 3$, are centrally symmetric, then $\Omega$ itself must also be centrally symmetric.)

1.5. As mentioned above, the Venkov-McMullen and Fuglede results imply not only that a convex polytope $\Omega \subset \mathbb{R}^{d}$ which can tile by translations is necessarily spectral, but also that $\Omega$ admits a lattice spectrum. Our approach allows us to establish that for certain convex polytopes, this spectrum is the unique one, up to translation.

First we have the following result in two dimensions:

Theorem 1.3. Let $\Omega$ be a centrally symmetric hexagon in $\mathbb{R}^{2}$. Then $\Omega$ has a unique spectrum up to translation.

This result is essentially contained in [IKT03], although it was not stated explicitly in that paper.

The three-dimensional version of the result is the following:

Theorem 1.4. Let $\Omega$ be a convex polytope in $\mathbb{R}^{3}$ which is spectral (and hence it can tile by translations), but which is neither a parallelepiped nor a hexagonal prism. Then $\Omega$ has a unique spectrum up to translation.

Remark that it is necessary in these results to exclude the parallelograms in $\mathbb{R}^{2}$, and the parallelepipeds and the centrally symmetric hexagonal prisms in $\mathbb{R}^{3}$. Indeed, these convex polytopes admit infinitely many non translation-equivalent spectra (see [JP99, Section 2]). 
1.6. The paper is organized as follows.

In Section 2 we present some preliminary background.

In Section 3 we give a proof of the fact that a spectral convex polytope $\Omega \subset \mathbb{R}^{d}$ must be centrally symmetric. The proof given is based on the argument from [KP02].

In Section 4 we prove that also all the facets of such an $\Omega$ are centrally symmetric (Theorem 1.1).

In Sections 50 we develop an approach to show that a spectral convex polytope $\Omega \subset \mathbb{R}^{d}$ can tile by translations. In Section 8 we give a proof, based on this approach, of the result that a spectral convex polygon $\Omega \subset \mathbb{R}^{2}$ can tile by translations.

The proof of the three-dimensional Theorem 1.2 is given through Sections 9615 ,

In Section 16 the results concerning the uniqueness of the spectrum up to translation are deduced (Theorems 1.3 and 1.4).

In the last Section 17 we give additional remarks and discuss some open problems.

\section{Preliminaries}

2.1. Notation. We fix some notation that will be used throughout the paper.

We shall denote by $\vec{e}_{1}, \ldots, \vec{e}_{d}$ the standard basis vectors in $\mathbb{R}^{d}$.

As usual, $\langle\cdot, \cdot\rangle$ and $|\cdot|$ are the Euclidean scalar product and norm in $\mathbb{R}^{d}$.

For a set $A \subset \mathbb{R}^{d}$ and a vector $x \in \mathbb{R}^{d}$, we use $\langle A, x\rangle$ to denote the set $\{\langle a, x\rangle: a \in A\}$.

We denote by $|\Omega|$ the Lebesgue measure of a measurable set $\Omega \subset \mathbb{R}^{d}$.

The Fourier transform in $\mathbb{R}^{d}$ will be normalized as

$$
\widehat{f}(\xi)=\int_{\mathbb{R}^{d}} f(x) e^{-2 \pi i\langle\xi, x\rangle} d x .
$$

2.2. Properties of spectra. We recall some basic properties of spectra that will be used in the paper.

Let $\Omega \subset \mathbb{R}^{d}$ be a bounded, measurable set of positive measure. A countable set $\Lambda \subset \mathbb{R}^{d}$ is a spectrum for $\Omega$ if the system of exponential functions $E(\Lambda)$ defined by (1.1) is an orthogonal basis in the space $L^{2}(\Omega)$. Since we have

$$
\left\langle e_{\lambda}, e_{\lambda^{\prime}}\right\rangle_{L^{2}(\Omega)}=\int_{\Omega} e^{-2 \pi i\left\langle\lambda^{\prime}-\lambda, x\right\rangle} d x=\widehat{\mathbb{1}}_{\Omega}\left(\lambda^{\prime}-\lambda\right),
$$

it follows that the orthogonality of $E(\Lambda)$ in $L^{2}(\Omega)$ is equivalent to the condition

$$
\Lambda-\Lambda \subset\left\{\widehat{\mathbb{1}}_{\Omega}=0\right\} \cup\{0\} .
$$

A set $\Lambda \subset \mathbb{R}^{d}$ is said to be uniformly discrete if there is $\delta>0$ such that $\left|\lambda^{\prime}-\lambda\right| \geqslant \delta$ for any two distinct points $\lambda, \lambda^{\prime}$ in $\Lambda$. The maximal constant $\delta$ with this property is called the separation constant of $\Lambda$, and will be denoted by $\delta(\Lambda)$.

The condition (2.1) implies that if $\Lambda$ is a spectrum for $\Omega$ then it is a uniformly discrete set, with separation constant $\delta(\Lambda)$ not smaller than

$$
\chi(\Omega):=\min \left\{|\xi|: \xi \in \mathbb{R}^{d}, \widehat{\mathbb{1}}_{\Omega}(\xi)=0\right\}>0 .
$$


It is easy to verify that the property of $\Lambda$ being a spectrum for $\Omega$ is invariant under translations of both $\Omega$ and $\Lambda$. It is also easy to check that if $\Lambda$ is a spectrum for $\Omega$, and if $A$ is an invertible $d \times d$ matrix, then the set $\left(A^{-1}\right)^{\top}(\Lambda)$ is a spectrum for $A(\Omega)$.

2.3. Limits of spectra. Let $\Lambda_{n}$ be a sequence of uniformly discrete sets in $\mathbb{R}^{d}$, such that $\delta\left(\Lambda_{n}\right) \geqslant \delta>0$. The sequence $\Lambda_{n}$ is said to converge weakly to a set $\Lambda$ if for every $\varepsilon>0$ and every $R$ there is $N$ such that

$$
\Lambda_{n} \cap B_{R} \subset \Lambda+B_{\varepsilon} \text { and } \Lambda \cap B_{R} \subset \Lambda_{n}+B_{\varepsilon}
$$

for all $n \geqslant N$, where by $B_{r}$ we denote the open ball of radius $r$ centered at the origin. In this case, the weak limit $\Lambda$ is also uniformly discrete, and moreover, $\delta(\Lambda) \geqslant \delta$.

By a standard diagonalization argument one can show that given any sequence $\Lambda_{n}$ satisfying $\delta\left(\Lambda_{n}\right) \geqslant \delta>0$, there is a subsequence $\Lambda_{n_{j}}$ which converges weakly to some (possibly empty) set $\Lambda$.

It is known that if for each $n$ the set $\Lambda_{n}$ is a spectrum for $\Omega$, and if $\Lambda_{n}$ converges weakly to a limit $\Lambda$, then also $\Lambda$ is a spectrum for $\Omega$. See, for example, [GL16, Section 3] where a simple proof of this fact can be found.

The latter fact easily implies that any spectrum $\Lambda$ of $\Omega$ must be a relatively dense set in $\mathbb{R}^{d}$, namely, there is $R>0$ such that every ball of radius $R$ intersects $\Lambda$. Moreover, the constant $R=R(\Omega)$ does not depend on the spectrum $\Lambda$. Indeed, if this was not true then there would exist a sequence $\Lambda_{n}$ of spectra for $\Omega$ which converges weakly to the empty set, which contradicts the fact that the weak limit must also be a spectrum for $\Omega$.

2.4. Fourier expansion with respect to a spectrum. If $\Lambda$ is a spectrum for $\Omega$, then each $f \in L^{2}(\Omega)$ admits a Fourier expansion with respect to the orthogonal basis $E(\Lambda)$. If we extend such a function $f$ to the whole $\mathbb{R}^{d}$ by defining it to be zero outside of $\Omega$, then we have $\left\langle f, e_{\lambda}\right\rangle_{L^{2}(\Omega)}=\widehat{f}(\lambda)$, hence the Fourier expansion of $f$ has the form

$$
f=\frac{1}{|\Omega|} \sum_{\lambda \in \Lambda} \widehat{f}(\lambda) e_{\lambda},
$$

and the series converges in $L^{2}(\Omega)$. Furthermore, Parseval's equality holds, namely

$$
\|f\|_{L^{2}(\Omega)}^{2}=\frac{1}{|\Omega|} \sum_{\lambda \in \Lambda}|\widehat{f}(\lambda)|^{2} .
$$

The following fact will be useful for us:

Lemma 2.1. For each function $f \in L^{2}(\Omega)$ (extended to be zero outside of $\Omega$ ) the series (2.3) converges unconditionally in $L^{2}$ on any bounded set to some measurable function $\tilde{f}$ defined a.e. on the whole $\mathbb{R}^{d}$, and $f$ coincides with $\tilde{f}$ a.e. on $\Omega$.

This is a simple consequence of the following:

Lemma 2.2. Let $\Lambda \subset \mathbb{R}^{d}$ be a uniformly discrete set, and $\{c(\lambda)\}$ be a sequence in $\ell^{2}(\Lambda)$. Then the series

$$
\sum_{\lambda \in \Lambda} c(\lambda) e_{\lambda}
$$

converges unconditionally in $L^{2}(S)$ for every bounded set $S \subset \mathbb{R}^{d}$. 
The latter fact is well-known, see for instance [You01, Section 4.3, Theorem 4] where it is proved in dimension one. For the reader's convenience we provide a self-contained proof in arbitrary dimension $d$.

Proof of Lemma 2.2. First we show that if $S$ is a bounded set then there is a constant $C=C(\Lambda, S)$, such that for every sequence $\{c(\lambda)\}$ with only finitely many non-zero terms we have

$$
\left\|\sum_{\lambda \in \Lambda} c(\lambda) e_{\lambda}\right\|_{L^{2}(S)}^{2} \leqslant C \sum_{\lambda \in \Lambda}|c(\lambda)|^{2} .
$$

Indeed, let $\delta>0$ denote the separation constant of $\Lambda$, and choose a smooth function $\varphi$ supported on a ball of radius $\delta / 2$ around the origin, such that $\int|\varphi(t)|^{2} d t=1$, and

$$
\eta:=\inf _{x \in S}|\widehat{\varphi}(x)|>0 .
$$

Then the left-hand side of (2.5) is not greater than $1 / \eta^{2}$ times

$$
\int_{\mathbb{R}^{d}}\left|\widehat{\varphi}(x) \sum_{\lambda \in \Lambda} c(\lambda) e_{\lambda}(x)\right|^{2} d x=\int_{\mathbb{R}^{d}}\left|\sum_{\lambda \in \Lambda} c(\lambda) \varphi(t+\lambda)\right|^{2} d t=\sum_{\lambda \in \Lambda}|c(\lambda)|^{2},
$$

hence (2.5) holds with $C=1 / \eta^{2}$.

Now it follows from (2.5) that given an arbitrary sequence $\{c(\lambda)\}$ in $\ell^{2}(\Lambda)$, the partial sums of the series (2.4) constitute a Cauchy sequence in $L^{2}(S)$ for every arrangement of the terms of the series, and the limit in $L^{2}(S)$ of these partial sums is the same for every such arrangement. This confirms the assertion of the lemma.

2.5. Convex polytopes. By a convex polytope $\Omega$ in $\mathbb{R}^{d}$ we mean a compact set which is the convex hull of a finite number of points. By a facet of $\Omega$ we refer to a $(d-1)$ dimensional face of $\Omega$, while a subfacet is a $(d-2)$-dimensional face.

If $G$ is a $k$-dimensional face of $\Omega(0 \leqslant k \leqslant d)$ then $|G|$ denotes the $k$-dimensional volume of $G$. For a facet $F$ of $\Omega$ we denote by $\sigma_{F}$ the surface measure on $F$.

The interior of $\Omega$ will be denoted by $\operatorname{int}(\Omega)$.

We say that $\Omega$ is centrally symmetric if there is a point $x \in \mathbb{R}^{d}$ (the center) such that $\Omega-x=-\Omega+x$. The following theorem, due to Minkowski, gives a criterion for the central symmetry of a convex polytope $\Omega$ in terms of the areas of its facets:

Theorem 2.3 (Minkowski). A convex polytope $\Omega$ is centrally symmetric if and only if for each facet $F$ of $\Omega$ there is a parallel facet $F^{\prime}$ such that $|F|=\left|F^{\prime}\right|$.

This is a consequence of the classical Minkowski's uniqueness theorem, see for example Gru07, Section 18.2].

We shall need some well-known facts about Fourier transforms related to convex polytopes in $\mathbb{R}^{d}$ (actually, in some of these results the convexity is not necessary). Since the proofs are not difficult, they are included for completeness.

Lemma 2.4. Let $\Omega$ be a convex polytope in $\mathbb{R}^{d}(d \geqslant 1)$. For each facet $F$ of $\Omega$, let $n_{F}$ denote the outward unit normal to $\Omega$ on $F$. Then

$$
-2 \pi i \xi \widehat{\mathbb{1}}_{\Omega}(\xi)=\sum n_{F} \widehat{\sigma}_{F}(\xi), \quad \xi \in \mathbb{R}^{d},
$$

where the sum is over all the facets $F$ of $\Omega$. 
Proof. Fix two vectors $\xi$ and $u$ in $\mathbb{R}^{d}$, and let

$$
\Phi(x):=u e^{-2 \pi i\langle\xi, x\rangle}, \quad x \in \mathbb{R}^{d} .
$$

Then we have

$$
\operatorname{div} \Phi(x)=-2 \pi i\langle\xi, u\rangle e^{-2 \pi i\langle\xi, x\rangle} .
$$

By the divergence theorem,

$$
\int_{\Omega} \operatorname{div} \Phi(x) d x=\int_{\partial \Omega}\langle\Phi(x), n(x)\rangle d \sigma(x)
$$

where $\sigma$ denotes the surface measure on the boundary $\partial \Omega$, and $n(x):=n_{F}$ if $x$ belongs to the relative interior of a facet $F$ of $\Omega$. This means that

$$
-2 \pi i\langle\xi, u\rangle \widehat{\mathbb{1}}_{\Omega}(\xi)=\sum\left\langle n_{F}, u\right\rangle \widehat{\sigma}_{F}(\xi),
$$

where the sum is over all the facets $F$ of $\Omega$. But since $\xi$ and $u$ were arbitrary vectors in $\mathbb{R}^{d}$, this implies (2.6).

Corollary 2.5. If $\Omega$ is a convex polytope in $\mathbb{R}^{d}(d \geqslant 1)$, then

$$
\left|\widehat{\mathbb{1}}_{\Omega}(\xi)\right| \leqslant \frac{|\partial \Omega|}{2 \pi} \cdot|\xi|^{-1}
$$

where $|\partial \Omega|$ denotes the total surface area of $\Omega$.

This follows from Lemma 2.4 using the fact that the right-hand side of (2.6) is bounded in norm by $|\partial \Omega|$.

Lemma 2.6. Let $\Omega$ be a convex polytope in $\mathbb{R}^{d}(d \geqslant 2)$, and $F$ be a facet of $\Omega$. Let $\theta(\xi, F)$ denote the angle between a non-zero vector $\xi \in \mathbb{R}^{d}$ and the outward normal vector to $\Omega$ on $F$. Then

$$
\left|\widehat{\sigma}_{F}(\xi)\right| \leqslant \frac{|\partial F|}{2 \pi} \cdot \frac{|\xi|^{-1}}{|\sin \theta(\xi, F)|},
$$

where $|\partial F|$ is the $(d-2)$-dimensional volume of the relative boundary of $F$.

Proof. By applying a rotation and a translation we may assume that $F$ is contained in the hyperplane $\left\{x_{1}=0\right\}$, and that the outward unit normal to $\Omega$ on $F$ is $\vec{e}_{1}$. Hence

$$
\widehat{\sigma}_{F}(\xi)=\varphi_{F}\left(\xi_{2}, \xi_{3}, \ldots, \xi_{d}\right),
$$

where $\varphi_{F}$ denotes the Fourier transform of the indicator function of the polytope in $\mathbb{R}^{d-1}$ obtained by projecting the facet $F$ on the $\left(x_{2}, x_{3}, \ldots, x_{d}\right)$ coordinates. Using Corollary 2.5, this implies that

$$
\left|\widehat{\sigma}_{F}(\xi)\right| \leqslant \frac{|\partial F|}{2 \pi}\left(\sum_{j=2}^{d} \xi_{j}^{2}\right)^{-1 / 2} .
$$

However, since we have

$$
\xi_{1}=\left\langle\xi, \vec{e}_{1}\right\rangle=|\xi| \cos \theta(\xi, F)
$$

it follows that

$$
\sum_{j=2}^{d} \xi_{j}^{2}=|\xi|^{2}-\xi_{1}^{2}=|\xi|^{2}\left(1-\cos ^{2} \theta(\xi, F)\right)=|\xi|^{2} \sin ^{2} \theta(\xi, F),
$$

so this proves the claim.

The previous lemmas imply the following result, that will be used in the next sections: 
Lemma 2.7. Let $\Omega$ be a convex polytope in $\mathbb{R}^{d}(d \geqslant 2)$. Assume that $A$ and $B$ are two parallel facets of $\Omega$, and that the outward unit normals to $\Omega$ on $A$ and $B$ are respectively the vectors $\vec{e}_{1}$ and $-\vec{e}_{1}$ (we also allow $A$ to be a facet which does not have a parallel facet, in which case we understand $B$ to be the empty set). Then there is $\alpha=\alpha(\Omega)>0$ such that

$$
-2 \pi i \xi_{1} \widehat{1}_{\Omega}(\xi)=\widehat{\sigma}_{A}(\xi)-\widehat{\sigma}_{B}(\xi)+O\left(\left|\xi_{1}\right|^{-1}\right), \quad\left|\xi_{1}\right| \rightarrow \infty
$$

in the cone

$$
K(\alpha):=\left\{\xi \in \mathbb{R}^{d}:\left|\xi_{j}\right| \leqslant \alpha\left|\xi_{1}\right| \quad(2 \leqslant j \leqslant d)\right\}
$$

Proof. By Lemma 2.4 we have

$$
-2 \pi i \xi_{1} \widehat{\mathbb{1}}_{\Omega}(\xi)=\widehat{\sigma}_{A}(\xi)-\widehat{\sigma}_{B}(\xi)+\sum\left\langle n_{F}, \vec{e}_{1}\right\rangle \widehat{\sigma}_{F}(\xi),
$$

where the sum is over all the facets $F$ of $\Omega$ other than $A$ and $B$. If $\alpha$ is sufficiently small, then the angle between any vector in $K(\alpha)$ and the outward normal to $\Omega$ on any facet $F$ other than $A$ and $B$, is bounded away from 0 and $\pi$. Hence by Lemma 2.6, the sum on the right-hand side of (2.9) is $O\left(|\xi|^{-1}\right)$ as $|\xi| \rightarrow \infty$ in the cone $K(\alpha)$. But since the ratio $\left|\xi_{1}\right| /|\xi|$ is bounded from below in $K(\alpha)$, this implies (2.7).

\section{SpeCtral CONVEX POLYTOpes ARE SyMmetriC}

3.1. In this section we give a proof of the following result:

Theorem 3.1 (Kolountzakis [Kol00]). Let $\Omega$ be a convex polytope in $\mathbb{R}^{d}(d \geqslant 2)$. If $\Omega$ is spectral then $\Omega$ is centrally symmetric.

In fact, it was proved in [Kol00] that any convex body (not assumed to be a polytope) which is spectral, must be centrally symmetric. This supports the conjecture that a spectral convex body $\Omega$ can tile by translations, as the central symmetry is a necessary condition for $\Omega$ to tile, by the Venkov-McMullen theorem.

There is another approach to prove Theorem 3.1, that was introduced in the paper KP02 due to Kolountzakis and Papadimitrakis. This approach is specific for polytopes, but on the other hand it does not require $\Omega$ to be convex. The main result in [KP02] gives a certain condition on a polytope $\Omega \subset \mathbb{R}^{d}$ that is necessary for its spectrality. If the polytope $\Omega$ is convex, then this condition coincides with the requirement that $\Omega$ is centrally symmetric.

For the completeness of our exposition, below we give a proof of Theorem 3.1 based on the argument in [KP02]. See also [Kol04, pp. 184-185]. The proof may also serve as a preparation for the next section, where the argument will be further developed.

3.2. Proof of Theorem 3.1. By Minkowski's Theorem 2.3 it would be enough to show that for each facet $A$ of $\Omega$ there is a parallel facet $B$ such that $|A|=|B|$. If this is not true, then there is a facet $A$ of $\Omega$ whose parallel facet $B$ satisfies $|A|>|B|$, where we understand $B$ to be the empty set if $A$ is a facet of $\Omega$ with no parallel facet.

By applying an affine transformation, we may assume that $A$ is contained in the hyperplane $\left\{x_{1}=0\right\}$, that $B$ is contained in the hyperplane $\left\{x_{1}=-1\right\}$, and that the 
outward unit normals to $\Omega$ on $A$ and $B$ are respectively the vectors $\vec{e}_{1}$ and $-\vec{e}_{1}$. It follows that

$$
\begin{aligned}
& \widehat{\sigma}_{A}(\xi)=\varphi_{A}\left(\xi_{2}, \xi_{3}, \ldots, \xi_{d}\right), \\
& \widehat{\sigma}_{B}(\xi)=e^{2 \pi i \xi_{1}} \varphi_{B}\left(\xi_{2}, \xi_{3}, \ldots, \xi_{d}\right),
\end{aligned}
$$

where $\varphi_{A}, \varphi_{B}$ are respectively the Fourier transforms of the indicator functions of the polytopes in $\mathbb{R}^{d-1}$ obtained by projecting the facets $A, B$ on the $\left(x_{2}, x_{3}, \ldots, x_{d}\right)$ coordinates. In particular, $\varphi_{A}$ and $\varphi_{B}$ are continuous functions, and

$$
\varphi_{A}(0)=|A|, \quad \varphi_{B}(0)=|B| .
$$

For any $r>0$ we denote by $S(r)$ the cylinder of radius $r$ along the $x_{1}$-axis, namely

$$
S(r):=\left\{t \vec{e}_{1}+w: t \in \mathbb{R}, w \in \mathbb{R}^{d},|w|<r\right\} .
$$

Notice that

$$
S(r)-S(r)=S(2 r)
$$

By assumption, we have $|A|>|B|$. Choose a number $\eta$ such that

$$
0<\eta<|A|-|B| \text {. }
$$

It follows from (3.1), (3.2) and (3.3) that there is $\varepsilon>0$ such that

$$
\left|\widehat{\sigma}_{A}(\xi)-\widehat{\sigma}_{B}(\xi)\right| \geqslant\left|\widehat{\sigma}_{A}(\xi)\right|-\left|\widehat{\sigma}_{B}(\xi)\right|>\eta, \quad \xi \in S(2 \varepsilon) .
$$

By Lemma 2.7 we have

$$
-2 \pi i \xi_{1} \widehat{\mathbb{1}}_{\Omega}(\xi)=\widehat{\sigma}_{A}(\xi)-\widehat{\sigma}_{B}(\xi)+O\left(\left|\xi_{1}\right|^{-1}\right), \quad\left|\xi_{1}\right| \rightarrow \infty
$$

in the cylinder $S(2 \varepsilon)$. It follows that there is $R>0$ such that

$$
\widehat{\mathbb{1}}_{\Omega}(\xi) \neq 0, \quad \xi \in S(2 \varepsilon) \backslash B_{R}
$$

where $B_{R}$ denotes the ball of radius $R$ centered at the origin.

Now let $\Lambda$ be a spectrum for $\Omega$. We claim that for any $\tau \in \mathbb{R}^{d}$, if $\lambda, \lambda^{\prime}$ are two points in $\Lambda \cap(S(\varepsilon)+\tau)$, then $\left|\lambda^{\prime}-\lambda\right| \leqslant R$. Indeed, if not then using (3.4) we get

$$
\lambda^{\prime}-\lambda \in S(2 \varepsilon) \backslash B_{R},
$$

but due to (3.5) this implies that $\widehat{\mathbb{1}}_{\Omega}\left(\lambda^{\prime}-\lambda\right) \neq 0$, a contradiction.

Since $\Lambda$ is a uniformly discrete set, it follows that $\Lambda \cap(S(\varepsilon)+\tau)$ is a finite set, for every $\tau \in \mathbb{R}^{d}$. Since $\Lambda$ is a relatively dense set, there is $M>0$ such that every ball of radius $M$ intersects $\Lambda$. The cylinder $S(M)$ may be covered by a finite number of cylinders $S(\varepsilon)+\tau_{j}(1 \leqslant j \leqslant N)$; hence $\Lambda \cap S(M)$ is also a finite set. But this implies that $S(M)$ must contain a ball of radius $M$ free from points of $\Lambda$, a contradiction. This completes the proof of Theorem 3.1 .

\section{Spectral COnVEX Polytopes have Symmetric FaCETS}

4.1. The result in Section 3 shows that the central symmetry is a necessary condition for a convex polytope $\Omega \subset \mathbb{R}^{d}$ to be spectral. In the present section we prove that also the central symmetry of all the facets of $\Omega$ is necessary for spectrality:

Theorem 4.1. Let $\Omega$ be a convex, centrally symmetric polytope in $\mathbb{R}^{d}(d \geqslant 3)$. If $\Omega$ is spectral then all the facets of $\Omega$ are also centrally symmetric. 
Recall that by the Venkov-McMullen theorem, the central symmetry of the facets is also a necessary condition for $\Omega$ to tile by translations. Hence this result further supports the conjecture that any spectral convex polytope $\Omega$ can tile by translations.

Notice that the conclusion cannot be further improved by showing that also all the $k$-dimensional faces of $\Omega$, for some $2 \leqslant k \leqslant d-2$, are centrally symmetric. Indeed, this would imply [McM70] that all the faces of $\Omega$ of every dimension are centrally symmetric. However, the 24-cell in $\mathbb{R}^{4}$ is a well-known example of a convex polytope which tiles by translations, and hence it is spectral, but which does not satisfy this property.

The rest of this section is devoted to the proof of Theorem 4.1. The proof is based on a development of the argument in [KP02].

4.2. Let $F$ be one of the facets of $\Omega$. As before, to prove that $F$ is centrally symmetric it would be enough, by Minkowski's Theorem 2.3 , to show that for each subfacet $A$ of $F$ there is a parallel subfacet $B$ of $F$ such that $|A|=|B|$. So, again, suppose to the contrary that $A, B$ are two parallel subfacets of $F$ such that $|A|>|B|$, with the agreement that $B$ is empty if $A$ has no parallel subfacet of $F$.

By applying an affine transformation, we may assume that

$$
\Omega=-\Omega,
$$

namely, $\Omega$ is symmetric about the origin,

$$
F \subset\left\{x_{1}=\frac{1}{2}\right\}
$$

and the outward unit normal to $\Omega$ on $F$ is $\vec{e}_{1}$,

$$
\begin{aligned}
& A \subset\left\{x_{1}=\frac{1}{2}, x_{2}=0\right\}, \\
& B \subset\left\{x_{1}=\frac{1}{2}, x_{2}=-1\right\},
\end{aligned}
$$

and the outward unit normals to $F$ on $A$ and $B$ are respectively $\vec{e}_{2}$ and $-\vec{e}_{2}$.

4.3. Let $\varphi_{F}$ (respectively, $\varphi_{A}$ and $\varphi_{B}$ ) denote the Fourier transform of the indicator function of the polytope in $\mathbb{R}^{d-1}$ (respectively $\mathbb{R}^{d-2}$ ) obtained by projecting the facet $F$ on the $\left(x_{2}, x_{3}, \ldots, x_{d}\right)$ coordinates (respectively, the subfacets $A$ and $B$ on the $\left(x_{3}, \ldots, x_{d}\right)$ coordinates $)$. Define

$$
\psi(\xi):=\operatorname{Re}\left[e^{-\pi i \xi_{1}}\left(\varphi_{A}\left(\xi_{3}, \ldots, \xi_{d}\right)-e^{2 \pi i \xi_{2}} \varphi_{B}\left(\xi_{3}, \ldots, \xi_{d}\right)\right)\right], \quad \xi \in \mathbb{R}^{d} .
$$

Also, for any three positive real numbers $L, \delta$ and $\alpha$, we let

$$
K(L, \delta, \alpha):=\left\{\xi \in \mathbb{R}^{d}: L \leqslant\left|\xi_{2}\right| \leqslant \delta\left|\xi_{1}\right|,\left|\xi_{j}\right| \leqslant \alpha\left|\xi_{2}\right|(3 \leqslant j \leqslant d)\right\} .
$$

Lemma 4.2. There is $\alpha>0$ such that given any $\eta>0$ one can find $\delta>0$ and $L$ such that

$$
\left|2 \pi^{2} \xi_{1} \xi_{2} \widehat{\mathbb{1}}_{\Omega}(\xi)+\psi(\xi)\right|<\eta, \quad \xi \in K(L, \delta, \alpha)
$$

Proof. Due to (4.1), the facet of $\Omega$ parallel to $F$ is $-F$. If $0<\delta \leqslant \alpha<1$, then the set $K(L, \delta, \alpha)$ is contained in the cone

$$
\left\{\left|\xi_{j}\right| \leqslant \alpha\left|\xi_{1}\right|, 2 \leqslant j \leqslant d\right\} .
$$

Hence by Lemma 2.7, if $\alpha$ is sufficiently small then

$$
-2 \pi i \xi_{1} \widehat{\mathbb{1}}_{\Omega}(\xi)=\widehat{\sigma}_{F}(\xi)-\widehat{\sigma}_{-F}(\xi)+O\left(\left|\xi_{1}\right|^{-1}\right), \quad\left|\xi_{1}\right| \rightarrow \infty
$$


in the cone (4.7). Observe that by (4.2) we have

$$
\widehat{\sigma}_{F}(\xi)-\widehat{\sigma}_{-F}(\xi)=2 i \operatorname{Im}\left[\widehat{\sigma}_{F}(\xi)\right]=2 i \operatorname{Im}\left[e^{-\pi i \xi_{1}} \varphi_{F}\left(\xi_{2}, \xi_{3}, \ldots, \xi_{d}\right)\right] .
$$

Now if $\xi \in K(L, \delta, \alpha)$ then the vector $\left(\xi_{2}, \xi_{3}, \ldots, \xi_{d}\right)$ belongs to the cone

$$
\left\{\left|\xi_{j}\right| \leqslant \alpha\left|\xi_{2}\right|, 3 \leqslant j \leqslant d\right\} \subset \mathbb{R}^{d-1},
$$

so again by Lemma 2.7 and by (4.3), (4.4) it follows that if $\alpha$ is sufficiently small then $-2 \pi i \xi_{2} \varphi_{F}\left(\xi_{2}, \xi_{3}, \ldots, \xi_{d}\right)=\varphi_{A}\left(\xi_{3}, \ldots, \xi_{d}\right)-e^{2 \pi i \xi_{2}} \varphi_{B}\left(\xi_{3}, \ldots, \xi_{d}\right)+O\left(\left|\xi_{2}\right|^{-1}\right), \quad\left|\xi_{2}\right| \rightarrow \infty$

in the cone (4.10). Combining (4.8), (4.9) and (4.11) shows that there is $\alpha>0$ and a positive constant $C$, such that for any $0<\delta \leqslant \alpha$ and any $L>0$ we have

$$
\left|2 \pi^{2} \xi_{1} \xi_{2} \widehat{\mathbb{1}}_{\Omega}(\xi)+\psi(\xi)\right| \leqslant C\left(\left|\xi_{2} / \xi_{1}\right|+\left|1 / \xi_{2}\right|\right), \quad \xi \in K(L, \delta, \alpha) .
$$

But for $\xi \in K(L, \delta, \alpha)$ we have $\left|\xi_{2} / \xi_{1}\right| \leqslant \delta$ and $\left|1 / \xi_{2}\right| \leqslant L^{-1}$. Hence given any $\eta>0$, by choosing $\delta$ sufficiently small and $L$ sufficiently large, we obtain (4.6).

4.4. Recall that, by assumption, we have $|A|>|B|$. Choose a number $\eta$ such that

$$
0<2 \eta<|A|-|B| \text {. }
$$

Use Lemma 4.2 to find $L, \delta$ and $\alpha$ such that (4.6) holds. Define the vector

$$
v_{\delta}:=2 \vec{e}_{1}+\delta \vec{e}_{2}=(2, \delta, 0,0, \ldots, 0) .
$$

For any $r>0$ we denote by $E(r, \delta)$ the union of balls of radius $r$ centered at the integral multiples of the vector $v_{\delta}$, that is,

$$
E(r, \delta):=\left\{k v_{\delta}+w: k \in \mathbb{Z}, w \in \mathbb{R}^{d},|w|<r\right\} .
$$

Notice that

$$
E(r, \delta)-E(r, \delta)=E(2 r, \delta)
$$

Since $\varphi_{A}, \varphi_{B}$ are continuous functions satisfying

$$
\varphi_{A}(0)=|A|, \quad \varphi_{B}(0)=|B|,
$$

it follows from (4.5), (4.12) and (4.13) that there is $\varepsilon>0$ such that

$$
\left|\psi(\xi)-\operatorname{Re}\left[|A|-e^{2 \pi i \xi_{2}}|B|\right]\right|<\eta, \quad \xi \in E(2 \varepsilon, \delta) .
$$

In particular, this implies that

$$
|\psi(\xi)| \geqslant|A|-|B|-\eta>\eta, \quad \xi \in E(2 \varepsilon, \delta) .
$$

4.5 .

Lemma 4.3. There is $R>0$ such that

$$
E(2 \varepsilon, \delta) \backslash B_{R} \subset K(L, \delta, \alpha),
$$

where $B_{R}$ denotes the ball of radius $R$ centered at the origin.

This can be verified easily, so we skip the proof. 
4.6. Now suppose that $\Lambda$ is a spectrum for $\Omega$. Use Lemma 4.3 to choose $R$ such that (4.16) holds. We claim that for any $\tau \in \mathbb{R}^{d}$, if $\lambda, \lambda^{\prime}$ are two points in $\Lambda \cap(E(\varepsilon, \delta)+\tau)$, then $\left|\lambda^{\prime}-\lambda\right| \leqslant R$. Indeed, if not then using (4.14) we get

$$
\lambda^{\prime}-\lambda \in E(2 \varepsilon, \delta) \backslash B_{R} \subset K(L, \delta, \alpha) .
$$

It thus follows from (4.6) and (4.15) that $\widehat{\mathbb{1}}_{\Omega}\left(\lambda^{\prime}-\lambda\right) \neq 0$, a contradiction.

Since $\Lambda$ is a uniformly discrete set, it follows that $\Lambda \cap(E(\varepsilon, \delta)+\tau)$ is a finite set, for every $\tau \in \mathbb{R}^{d}$. Since $\Lambda$ is a relatively dense set, there is $M>0$ such that every ball of radius $M$ intersects $\Lambda$. Let $S(M, \delta)$ denote the cylinder of radius $M$ along the vector $v_{\delta}$,

$$
S(M, \delta):=\left\{t v_{\delta}+w: t \in \mathbb{R}, w \in \mathbb{R}^{d},|w|<M\right\} .
$$

Then $S(M, \delta)$ may be covered by a finite number of sets $E(\varepsilon, \delta)+\tau_{j}(1 \leqslant j \leqslant N)$; hence $\Lambda \cap S(M, \delta)$ is also a finite set. It follows that $S(M, \delta)$ contains a ball of radius $M$ free from points of $\Lambda$, a contradiction. This completes the proof of Theorem 4.1 .

\section{COVERING AND PACKING}

It was shown in Sections 3 and 4 that if a convex polytope $\Omega \subset \mathbb{R}^{d}$ is spectral, then it must be centrally symmetric and have centrally symmetric facets. In order to prove that $\Omega$ tiles by translations, a conceivable strategy may therefore be to try and show that every belt of $\Omega$ must consist of either 4 or 6 facets. Indeed, this would imply that $\Omega$ tiles, by the Venkov-McMullen theorem.

Our approach, however, will not be based on such a strategy. Instead, we will use another condition, given in terms of the spectrum $\Lambda$, which implies that $\Omega$ tiles by translations. In this section, we prove the sufficiency of this condition (Corollary 5.3).

5.1. Let $\Omega \subset \mathbb{R}^{d}$ be a convex polytope, which is centrally symmetric and has centrally symmetric facets. If $F$ is any facet of $\Omega$, then by the central symmetry, the opposite facet $F^{\prime}$ is a translate of $F$. We shall denote by $\tau_{F}$ the translation vector in $\mathbb{R}^{d}$ which carries $F^{\prime}$ onto $F$.

Following [Ven54, McM80] we consider the set

$$
T=T(\Omega)=\left\{\sum_{F} k_{F} \tau_{F}: k_{F} \in \mathbb{Z}\right\},
$$

that is, $T$ is the set of all linear combinations with integer coefficients of the vectors $\tau_{F}$, where $F$ goes through all the facets of $\Omega$. The set $T$ is a countable subgroup of $\mathbb{R}^{d}$.

Theorem 5.1 ([Ven54, McM80]). $\Omega+T$ is a covering, that is, each point in $\mathbb{R}^{d}$ belongs to at least one of the sets $\Omega+\tau, \tau \in T$.

This is a part of the Venkov-McMullen theorem, which characterizes the convex bodies that tile by translations by the four conditions (i) (iv) mentioned in Section 1.2. In the sufficiency part of the theorem it is shown that these four conditions imply that $\Omega+T$ is a tiling. However the last condition, namely the requirement (iv) that each belt consists of exactly 4 or 6 facets, is not used in that part of the proof where it is shown that $\Omega+T$ is a covering (see [McM80, pp. 115-116] where the latter fact is also mentioned explicitly). Hence the proof yields that the first three conditions (i) (iii) are enough to conclude that $\Omega+T$ is a covering, as stated in Theorem 5.1 . 
Observe that Theorem 5.1 implies that $T$ is a relatively dense set in $\mathbb{R}^{d}$.

It also follows from this theorem that, in order to prove that $\Omega$ tiles by translations, it would be enough to show that $\Omega+T$ is a packing, which means that the sets $\Omega+\tau$, $\tau \in T$, are disjoint up to measure zero. Indeed, in such a case $\Omega+T$ is simultaneously a covering and a packing, hence $\Omega$ tiles by translations along the set $T$.

Notice that if $\Omega+T$ is a packing (and hence a tiling), then $T$ must be a uniformly discrete set in $\mathbb{R}^{d}$. So in this case $T$ is a subgroup of $\mathbb{R}^{d}$ which is both uniformly discrete and relatively dense, and it follows that $T$ must be a lattice. As mentioned in [McM80, the tiling by translations of $\Omega$ along the lattice $T$ constitutes a face-to-face tiling.

5.2. The next lemma gives a sufficient condition for $\Omega+T$ to be a packing:

Lemma 5.2. Suppose that $\Lambda \subset \mathbb{R}^{d}$ is a set satisfying the following condition:

$$
\left\langle\Lambda-\Lambda, \tau_{F}\right\rangle \subset \mathbb{Z}
$$

for every facet $F$ of $\Omega$. If the system of exponentials $E(\Lambda)$ is complete in $L^{2}(\Omega)$, then $\Omega+T$ is a packing.

Proof. By translating $\Lambda$ we may assume that it contains the origin, hence $\left\langle\Lambda, \tau_{F}\right\rangle \subset \mathbb{Z}$ for every facet $F$. It follows that the exponential functions $e_{\lambda}(\lambda \in \Lambda)$ are periodic with respect to $T$, namely

$$
e_{\lambda}(x+\tau)=e_{\lambda}(x)
$$

for every $\tau \in T$. If $\Omega+T$ is not a packing then there exist distinct vectors $\tau^{\prime}, \tau^{\prime \prime} \in T$ such that the set $\left(\Omega+\tau^{\prime}\right) \cap\left(\Omega+\tau^{\prime \prime}\right)$ has positive measure. Thus the set $E$ defined by

$$
E:=\Omega \cap(\Omega-\tau), \quad \tau:=\tau^{\prime \prime}-\tau^{\prime}
$$

is a set of positive measure, and $E, E+\tau$ are both contained in $\Omega$. Hence the function $f:=\mathbb{1}_{E}-\mathbb{1}_{E+\tau}$ is supported by $\Omega$, and since $\tau \neq 0$, the function $f$ does not vanish identically a.e. On the other hand, for every $\lambda \in \Lambda$ we have

$$
\left\langle e_{\lambda}, f\right\rangle_{L^{2}(\Omega)}=\int_{E} e_{\lambda}(x) d x-\int_{E+\tau} e_{\lambda}(x) d x=0,
$$

due to the periodicity of $e_{\lambda}$. Hence $f$ is orthogonal in $L^{2}(\Omega)$ to all the exponentials $\left\{e_{\lambda}\right\}$, $\lambda \in \Lambda$, which contradicts the completeness of the system $E(\Lambda)$ in the space $L^{2}(\Omega)$.

5.3. Combining Theorem 5.1 and Lemma 5.2 we obtain the following:

Corollary 5.3. Let $\Omega \subset \mathbb{R}^{d}$ be a convex polytope, which is centrally symmetric and has centrally symmetric facets. Suppose that $\Omega$ admits a spectrum $\Lambda$ satisfying (5.2) for every facet $F$ of $\Omega$. Then $\Omega+T$ is a tiling, and so $\Omega$ can tile by translations.

Moreover, in this case the set $T$ defined by (5.1) is a lattice in $\mathbb{R}^{d}$, and $\Omega$ tiles faceto-face by translations along the lattice $T$.

Remark. The formulation of Corollary 5.3 is inspired by [IKT03, p. 568], where the assertion was proved in dimension $d=2$ by directly showing that $\Omega$ must be either a parallelogram or a centrally symmetric hexagon. The proof in arbitrary dimension that we have given above is based on different considerations than the one in [KT03. 


\section{Structure of spectrum, I}

We obtained in Section 5 a sufficient condition for a spectral convex polytope $\Omega$ in $\mathbb{R}^{d}$ to tile by translations. This condition (Corollary 5.3) requires the existence of a spectrum $\Lambda$ admitting a certain structure. In the present section we start to develop an approach to analyze the structure of a given spectrum $\Lambda$.

6.1. Let $\Omega \subset \mathbb{R}^{d}$ be a convex polytope, which is centrally symmetric and has centrally symmetric facets. We will assume that $\Omega=-\Omega$, that is, $\Omega$ is symmetric about the origin. Let $F$ be one of the facets of $\Omega$, and assume that $F \subset\left\{x_{1}=\frac{1}{2}\right\}$, and that the center of $F$ is the point $\left(\frac{1}{2}, 0,0, \ldots, 0\right)$.

These assumptions are made merely for convenience. Later on, we will reduce the general situation to this more specific one by applying an affine transformation.

The assumptions imply that

$$
F=\left\{\frac{1}{2}\right\} \times \Sigma
$$

where $\Sigma$ is a convex polytope in $\mathbb{R}^{d-1}$ such that

$$
\Sigma=-\Sigma \text {. }
$$

The facet opposite to $F$ is therefore

$$
-F=\left\{-\frac{1}{2}\right\} \times \Sigma .
$$

6.2. For $\alpha>0$ we consider the cone

$$
K(\alpha):=\left\{\xi \in \mathbb{R}^{d}:\left|\xi_{j}\right| \leqslant \alpha\left|\xi_{1}\right| \quad(2 \leqslant j \leqslant d)\right\} .
$$

Lemma 6.1. There is $\alpha=\alpha(\Omega)>0$ such that

$$
\pi \xi_{1} \widehat{\mathbb{1}}_{\Omega}(\xi)=\sin \pi \xi_{1} \cdot \widehat{\mathbb{1}}_{\Sigma}\left(\xi_{2}, \xi_{3}, \ldots, \xi_{d}\right)+O\left(\left|\xi_{1}\right|^{-1}\right), \quad\left|\xi_{1}\right| \rightarrow \infty
$$

in the cone $K(\alpha)$.

Proof. By Lemma 2.7, if $\alpha$ is sufficiently small then

$$
-2 \pi i \xi_{1} \widehat{\mathbb{1}}_{\Omega}(\xi)=\widehat{\sigma}_{F}(\xi)-\widehat{\sigma}_{-F}(\xi)+O\left(\left|\xi_{1}\right|^{-1}\right), \quad\left|\xi_{1}\right| \rightarrow \infty
$$

in $K(\alpha)$. But we have

$$
\widehat{\sigma}_{F}(\xi)=e^{-\pi i \xi_{1}} \widehat{\mathbb{1}}_{\Sigma}\left(\xi_{2}, \xi_{3}, \ldots, \xi_{d}\right) \text { and } \widehat{\sigma}_{-F}(\xi)=e^{\pi i \xi_{1}} \widehat{\mathbb{1}}_{\Sigma}\left(\xi_{2}, \xi_{3}, \ldots, \xi_{d}\right),
$$

which yields the conclusion of the lemma.

6.3. Assume now that we are given a set $\Lambda \subset \mathbb{R}^{d}$ which is a spectrum for $\Omega$. To this spectrum $\Lambda$ we associate a set $\Pi \subset \mathbb{R}^{d-1}$ defined as follows: $\Pi$ is the set of all points $s \in \mathbb{R}^{d-1}$ such that for every open ball $B$ containing $s$, the cylinder $\mathbb{R} \times B$ contains infinitely many points of $\Lambda$.

If we denote a point in $\mathbb{R}^{d}$ as $(t, s) \in \mathbb{R} \times \mathbb{R}^{d-1}$, then one can check that a point $s \in \mathbb{R}^{d-1}$ belongs to $\Pi$ if and only if there is a sequence $\left(t_{n}, s_{n}\right) \in \Lambda$ such that

$$
\left|t_{n}\right| \rightarrow \infty, \quad s_{n} \rightarrow s \quad(n \rightarrow \infty)
$$

It is also not difficult to verify that $\Pi$ is a closed subset of $\mathbb{R}^{d-1}$.

The motivation for introducing the set $\Pi$ is the following observation: 
Lemma 6.2. For each $s \in \Pi$ there is a (unique) number $0 \leqslant \theta(s)<1$ such that

$$
\Lambda \cap\left(\mathbb{R} \times\left(s+U_{\Sigma}\right)\right) \subset(\mathbb{Z}+\theta(s)) \times \mathbb{R}^{d-1},
$$

where

$$
U_{\Sigma}:=\left\{\widehat{\mathbb{1}}_{\Sigma} \neq 0\right\}
$$

In other words, if $\left(t^{\prime}, s^{\prime}\right) \in \Lambda$ and if $\widehat{\mathbb{1}}_{\Sigma}\left(s^{\prime}-s\right) \neq 0$, then $t^{\prime} \in \mathbb{Z}+\theta(s)$.

Proof. It would be enough to show that if $\left(t^{\prime}, s^{\prime}\right)$ and $\left(t^{\prime \prime}, s^{\prime \prime}\right)$ are two points in $\Lambda \cap(\mathbb{R} \times$ $\left.\left(s+U_{\Sigma}\right)\right)$, then $t^{\prime \prime}-t^{\prime} \in \mathbb{Z}$. Since $s \in \Pi$ there is a sequence $\left(t_{n}, s_{n}\right) \in \Lambda$ such that $\left|t_{n}\right| \rightarrow \infty, s_{n} \rightarrow s$. The vectors $\left(t^{\prime}-t_{n}, s^{\prime}-s_{n}\right)$ and $\left(t^{\prime \prime}-t_{n}, s^{\prime \prime}-s_{n}\right)$ belong to the set $(\Lambda-\Lambda) \backslash\{0\}$ for all large enough $n$, hence they lie in the zero set of $\widehat{\mathbb{1}}_{\Omega}$. Using Lemma 6.1 it follows that

$$
\sin \pi\left(t^{\prime}-t_{n}\right) \cdot \widehat{\mathbb{1}}_{\Sigma}\left(s^{\prime}-s_{n}\right) \rightarrow 0, \quad \sin \pi\left(t^{\prime \prime}-t_{n}\right) \cdot \widehat{\mathbb{1}}_{\Sigma}\left(s^{\prime \prime}-s_{n}\right) \rightarrow 0,
$$

as $n \rightarrow \infty$. Recall that $s^{\prime}-s$ and $s^{\prime \prime}-s$ are not in the zero set of $\widehat{\mathbb{1}}_{\Sigma}$. Hence $\left|\widehat{\mathbb{1}}_{\Sigma}\left(s^{\prime}-s_{n}\right)\right|$ and $\left|\widehat{\mathbb{1}}_{\Sigma}\left(s^{\prime \prime}-s_{n}\right)\right|$ remain bounded away from zero as $n \rightarrow \infty$. We conclude that

$$
\sin \pi\left(t^{\prime}-t_{n}\right), \quad \sin \pi\left(t^{\prime \prime}-t_{n}\right)
$$

both tend to zero as $n \rightarrow \infty$, or equivalently,

$$
\operatorname{dist}\left(t^{\prime}-t_{n}, \mathbb{Z}\right), \quad \operatorname{dist}\left(t^{\prime \prime}-t_{n}, \mathbb{Z}\right)
$$

both tend to zero. But

$$
\operatorname{dist}\left(t^{\prime \prime}-t^{\prime}, \mathbb{Z}\right) \leqslant \operatorname{dist}\left(t^{\prime}-t_{n}, \mathbb{Z}\right)+\operatorname{dist}\left(t^{\prime \prime}-t_{n}, \mathbb{Z}\right),
$$

which implies that $t^{\prime \prime}-t^{\prime} \in \mathbb{Z}$.

Corollary 6.3. Let $s^{\prime}, s^{\prime \prime} \in \Pi$. If $\theta\left(s^{\prime}\right) \neq \theta\left(s^{\prime \prime}\right)$, then $\widehat{\mathbb{1}}_{\Sigma}\left(s^{\prime \prime}-s^{\prime}\right)=0$.

Proof. Let $\left(t_{n}, s_{n}\right) \in \Lambda$ be a sequence such that $\left|t_{n}\right| \rightarrow \infty, s_{n} \rightarrow s^{\prime \prime}$. If $\widehat{\mathbb{1}}_{\Sigma}\left(s^{\prime \prime}-s^{\prime}\right) \neq 0$, then for large enough $n$ we would have $\widehat{\mathbb{1}}_{\Sigma}\left(s_{n}-s^{\prime}\right) \neq 0$. By Lemma 6.2 it follows that $t_{n} \in \mathbb{Z}+\theta\left(s^{\prime}\right)$. On the other hand, for all large enough $n$ we also have $\widehat{\mathbb{1}}_{\Sigma}\left(s_{n}-s^{\prime \prime}\right) \neq 0$, since

$$
\widehat{\mathbb{1}}_{\Sigma}(0)=|\Sigma|>0
$$

Hence, again by Lemma 6.2, we have $t_{n} \in \mathbb{Z}+\theta\left(s^{\prime \prime}\right)$. So we must have $\theta\left(s^{\prime}\right)=\theta\left(s^{\prime \prime}\right)$.

6.4. Lemma 6.2 allows us to define an equivalence relation on $\Pi$ by saying that $s^{\prime} \sim s^{\prime \prime}$ if $\theta\left(s^{\prime}\right)=\theta\left(s^{\prime \prime}\right)$. It follows from Corollary 6.3 that if $\Pi^{\prime}$ and $\Pi^{\prime \prime}$ are two distinct equivalence classes, then

$$
\Pi^{\prime \prime}-\Pi^{\prime} \subset\left\{\widehat{\mathbb{1}}_{\Sigma}=0\right\} .
$$

The set $\left\{\widehat{\mathbb{1}}_{\Sigma}=0\right\}$ is disjoint from the open ball of radius $\chi(\Sigma)>0$ centered at the origin, see (2.2). It follows that each equivalence class is a closed set, and that there can be at most countably many equivalence classes. So we may enumerate them as $\Pi_{0}, \Pi_{1}, \Pi_{2}, \ldots$ (finitely or infinitely many), and we denote by $\theta_{0}, \theta_{1}, \theta_{2}, \ldots$ respectively the values of the function $\theta(s)$ on these equivalence classes. 
6.5. To illustrate the construction above, let us consider two representative examples.

Example 6.4. Assume that $\Omega$ tiles face-to-face along a lattice $T$ of translation vectors, which in this case is given by (5.1). Since the facet $F$ has the form $F=\left\{\frac{1}{2}\right\} \times \Sigma$, we have $\tau_{F}=(1,0,0, \ldots, 0) \in T$. Let $\Lambda$ be a spectrum of $\Omega$ given by the dual lattice, that is, $\Lambda=T^{*}$. Then $\langle\Lambda, \tau\rangle \subset \mathbb{Z}$ for any $\tau \in T$. In particular this is true for $\tau=\tau_{F}$, hence

$$
\Lambda \subset \mathbb{Z} \times \mathbb{R}^{d-1}
$$

It follows that $\theta(s)=0$ for all $s \in \Pi$. Thus in this case the set $\Pi$ consists of a single equivalence class, namely $\Pi=\Pi_{0}$, and we have $\theta_{0}=0$.

Example 6.5. Assume that $\Omega=I \times \Sigma$, where $I$ denotes the interval $\left[-\frac{1}{2}, \frac{1}{2}\right]$. Then $\Omega$ is a prism with base $\Sigma$. Suppose that $\Sigma$ is a spectral set, and let $\Gamma \subset \mathbb{R}^{d-1}$ be a spectrum for $\Sigma$. For each $\gamma \in \Gamma$, let $\theta(\gamma)$ be an arbitrary real number, $0 \leqslant \theta(\gamma)<1$, and define

$$
\Lambda:=\bigcup_{\gamma \in \Gamma}(\mathbb{Z}+\theta(\gamma)) \times\{\gamma\}
$$

It is known, see [JP99, Theorem 4], that $\Lambda$ is a spectrum for $\Omega$. In this case we clearly have $\Pi=\Gamma$, and the numbers $\theta(\gamma)$ coincide with the ones given by Lemma 6.2. The equivalence classes $\Pi_{j}$ depend on the specific choice of the numbers $\theta(\gamma)$, but in the case when all the $\theta(\gamma)$ are distinct, the sets $\Pi_{j}$ are singletons. Observe that we have

$$
\Pi_{j}-\Pi_{k} \subset\left\{\widehat{\mathbb{1}}_{\Sigma}=0\right\} \quad(k \neq j),
$$

since $\Gamma$ is a spectrum for $\Sigma$. This is in accordance with Corollary 6.3,

\section{Structure of Spectrum, II}

In this section we continue to work under the same assumptions as in Section 6 , Namely, we assume that $\Omega \subset \mathbb{R}^{d}$ is a convex polytope which is centrally symmetric, $\Omega=-\Omega$, and has centrally symmetric facets, $F$ is one of the facets of $\Omega$, and $F=\left\{\frac{1}{2}\right\} \times \Sigma$ where $\Sigma$ is a convex polytope in $\mathbb{R}^{d-1}$ such that $\Sigma=-\Sigma$.

We also assume that $\Lambda$ is a spectrum for $\Omega$, and to this spectrum $\Lambda$ we associate the set $\Pi \subset \mathbb{R}^{d-1}$ that was defined in Section 6 .

7.1. From the given spectrum $\Lambda$ one can construct a new spectrum $\Lambda^{\prime}$ for $\Omega$, in the following way. Consider the sequence of translates of $\Lambda$ given by

$$
\Lambda-k \cdot(1,0,0, \ldots, 0), \quad k=1,2,3, \ldots
$$

Each one of these sets is a spectrum for $\Omega$, and they are uniformly discrete with the same separation constant. Hence one may extract from this sequence a subsequence

$$
\Lambda-k_{n} \cdot(1,0,0, \ldots, 0), \quad k_{n} \rightarrow \infty,
$$

which converges weakly to some set $\Lambda^{\prime}$, which is also a spectrum for $\Omega$ (see Section 2.3). Notice that we do not make any claim concerning the uniqueness of the weak limit $\Lambda^{\prime}$, which in general may depend on the particular subsequence that was selected.

Lemma 7.1. We have

$$
\Lambda^{\prime} \subset \bigcup_{j \geqslant 0}\left(\mathbb{Z}+\theta_{j}\right) \times \Pi_{j}
$$


We remind that by $\theta_{j}(j \geqslant 0)$ we denote the distinct values attained by the function $\theta(s)$ defined on $\Pi$, given in Lemma 6.2, and

$$
\Pi_{j}=\left\{s \in \Pi: \theta(s)=\theta_{j}\right\} .
$$

Recall also that according to Corollary 6.3 we have

$$
\Pi_{k}-\Pi_{j} \subset\left\{\widehat{\mathbb{1}}_{\Sigma}=0\right\} \quad(j \neq k),
$$

hence Lemma 7.1 reveals a certain structure satisfied by the new spectrum $\Lambda^{\prime}$.

Proof of Lemma 7.1. The claim is equivalent to the statement that for every $\left(t^{\prime}, s^{\prime}\right) \in \Lambda^{\prime}$ we have $s^{\prime} \in \Pi$ and $t^{\prime} \in \mathbb{Z}+\theta\left(s^{\prime}\right)$. Let therefore $\left(t^{\prime}, s^{\prime}\right) \in \Lambda^{\prime}$. Since $\Lambda^{\prime}$ is the weak limit of the sequence $\Lambda-k_{n} \cdot(1,0,0, \ldots, 0)$, there exist $\left(t_{n}, s_{n}\right) \in \Lambda$ such that

$$
\left(t_{n}-k_{n}, s_{n}\right) \rightarrow\left(t^{\prime}, s^{\prime}\right), \quad n \rightarrow \infty .
$$

Hence $s_{n} \rightarrow s^{\prime}$, and $t_{n} \rightarrow \infty$ since $k_{n} \rightarrow \infty$. This implies that $s^{\prime} \in \Pi$. For all sufficiently large $n$ we have $\widehat{\mathbb{1}}_{\Sigma}\left(s_{n}-s^{\prime}\right) \neq 0$, thus by Lemma 6.2 we have $t_{n} \in \mathbb{Z}+\theta\left(s^{\prime}\right)$. Since $t_{n}-k_{n} \rightarrow t^{\prime}$ and the $k_{n}$ are integers, this implies that also $t^{\prime} \in \mathbb{Z}+\theta\left(s^{\prime}\right)$.

7.2. Given a point $\left(t_{0}, s_{0}\right)$ in $\mathbb{R} \times \mathbb{R}^{d-1}$, we associate with it a function $f$ defined by

$$
f(x, y):=\mathbb{1}_{I}(x) e^{2 \pi i t_{0} x} \mathbb{1}_{\Sigma}(y) e^{2 \pi i\left\langle s_{0}, y\right\rangle}, \quad(x, y) \in \mathbb{R} \times \mathbb{R}^{d-1}
$$

where $I$ denotes again the interval $\left[-\frac{1}{2}, \frac{1}{2}\right]$. Notice that the function $f$ is supported by the prism $I \times \Sigma$. This prism is contained in $\Omega$, since $\left\{\frac{1}{2}\right\} \times \Sigma$ and $\left\{-\frac{1}{2}\right\} \times \Sigma$ are facets of $\Omega$, and $\Omega$ is convex. Hence $f$ is also supported by $\Omega$.

It follows from the definition (17.4) of $f$ that its Fourier transform is given by

$$
\widehat{f}(t, s)=\widehat{\mathbb{1}}_{I}\left(t-t_{0}\right) \widehat{\mathbb{1}}_{\Sigma}\left(s-s_{0}\right), \quad(t, s) \in \mathbb{R} \times \mathbb{R}^{d-1} .
$$

Using the function $f$ thus defined, we can prove a result similar to Lemma 7.1 but which is concerned with the originally given spectrum $\Lambda$. However, the conclusion is somewhat weaker, as the right hand side of (7.1) is replaced by a larger set:

Lemma 7.2. We have

$$
\Lambda \subset \bigcup_{j \geqslant 0}\left(\mathbb{Z}+\theta_{j}\right) \times\left(\Pi_{j}+U_{\Sigma}\right)
$$

where, as before, we let

$$
U_{\Sigma}=\left\{\widehat{\mathbb{1}}_{\Sigma} \neq 0\right\}
$$

Proof. By Lemma 6.2 we have

$$
\Lambda \cap\left(\mathbb{R} \times\left(\Pi_{j}+U_{\Sigma}\right)\right) \subset\left(\mathbb{Z}+\theta_{j}\right) \times\left(\Pi_{j}+U_{\Sigma}\right)
$$

for every $j$. Hence, to prove the claim it would be enough to show that the sets $\Pi_{j}+U_{\Sigma}$ cover the whole $\mathbb{R}^{d-1}$. Suppose to the contrary that there is a point $s_{0} \in \mathbb{R}^{d-1}$ which lies outside all the sets $\Pi_{j}+U_{\Sigma}$. Since $U_{\Sigma}=-U_{\Sigma}$, this means that

$$
\widehat{\mathbb{1}}_{\Sigma}\left(s-s_{0}\right)=0, \quad s \in \Pi \text {. }
$$

Let $t_{0}$ be an arbitrary real number, and consider the function $f$ defined by (17.4). Then $f$ is supported by $\Omega$, and by (7.5) its Fourier transform $\widehat{f}$ vanishes on $\mathbb{R} \times \Pi$. In particular we have $\widehat{f}(\lambda)=0$ for all $\lambda \in \Lambda^{\prime}$, due to Lemma 7.1. That is,

$$
\left\langle f, e_{\lambda}\right\rangle_{L^{2}(\Omega)}=\widehat{f}(\lambda)=0, \quad \lambda \in \Lambda^{\prime} .
$$


Hence $f$ is orthogonal in $L^{2}(\Omega)$ to all the exponentials $\left\{e_{\lambda}\right\}, \lambda \in \Lambda^{\prime}$, which contradicts the completeness of the system $E\left(\Lambda^{\prime}\right)$ in the space $L^{2}(\Omega)$. This concludes the proof.

Corollary 7.3. Assume that the function $\theta(s)$ is constant on $\Pi$. Then

$$
\Lambda-\Lambda \subset \mathbb{Z} \times \mathbb{R}^{d-1}
$$

Proof. It is assumed that $\Pi=\Pi_{0}$ and $\theta(s)=\theta_{0}$ for all $s \in \Pi$. Hence by Lemma 7.2 , the set $\Lambda$ is contained in $\left(\mathbb{Z}+\theta_{0}\right) \times\left(\Pi_{0}+U_{\Sigma}\right)$, which implies (7.6).

7.3. Corollary 7.3 is an important point in our approach to the proof that $\Omega$ can tile by translations. Let us clarify its role. Recall that a sufficient condition for $\Omega$ to tile was given by Corollary [5.3, namely, it is enough to know that the spectrum $\Lambda$ satisfies condition (5.2) for every facet $F$ of $\Omega$. For the facet $F=\left\{\frac{1}{2}\right\} \times \Sigma$ we have $\tau_{F}=(1,0,0, \ldots, 0)$, hence for this facet the condition (5.2) is the same as (77.6). It thus follows from Corollary 7.3 that in order to establish (5.2) for the facet $F=\left\{\frac{1}{2}\right\} \times \Sigma$, it would be sufficient to prove that the function $\theta(s)$ is constant on $\Pi$.

\section{Spectral Convex polygons tile the Plane}

8.1. In this section we will demonstrate how the tools developed so far can be useful in our problem, by showing that at this point they already enable us to give an alternative proof of the following result in dimension $d=2$ :

Theorem 8.1 (Iosevich, Katz and Tao [IKT03]). Let $\Omega$ be a convex polygon in $\mathbb{R}^{2}$. If $\Omega$ is spectral, then $\Omega$ tiles by translations.

We remark that the paper [IKT03] actually contains a proof of a more general result, which yields the same conclusion for any convex body $\Omega \subset \mathbb{R}^{2}$ (not assumed a priori to be a polygon).

8.2. In order to prove Theorem 8.1, we now restrict ourselves to dimension $d=2$. Let $\Omega$ be a convex polygon in $\mathbb{R}^{2}$. Assume that $\Omega$ is spectral, and let $\Lambda$ be a spectrum for $\Omega$. We must prove that $\Omega$ tiles by translations. This is obvious if $\Omega$ is a parallelogram, so in what follows we will assume that $\Omega$ is not a parallelogram.

By Theorem 3.1 the polygon $\Omega$ is centrally symmetric, and since the facets of $\Omega$ are line segments, then automatically also all the facets of $\Omega$ are centrally symmetric.

Lemma 8.2. Let $\Omega$ be a convex, centrally symmetric polygon in $\mathbb{R}^{2}$, and assume that $\Omega$ is not a parallelogram. If $\Lambda$ is a spectrum of $\Omega$, then

$$
\left\langle\Lambda-\Lambda, \tau_{F}\right\rangle \subset \mathbb{Z}
$$

for every facet $F$ of $\Omega$.

Theorem 8.1 follows immediately from a combination of Lemma 8.2 and Corollary 5.3 , Hence, it only remains to prove the lemma.

Lemma 8.2 was proved in [IKT03, Proposition 3.1], and was also used there to deduce that $\Omega$ tiles by translations. However, both our proof of Lemma 8.2 , and the argument we use to deduce Theorem 8.1 from Lemma 8.2, are different from those in [IKT03]. 


\subsection{Now we give our proof of Lemma 8.2.}

Proof of Lemma 8.2. Let $F$ be a facet of $\Omega$. We must show that if $\Lambda$ is a spectrum of $\Omega$, then it satisfies condition (8.1). By applying an affine transformation we may assume that $\Omega$ is symmetric about the origin, $\Omega=-\Omega$, and that $F=\left\{\frac{1}{2}\right\} \times I$, where $I$ is the interval $\left[-\frac{1}{2}, \frac{1}{2}\right]$. Hence we have $\Sigma=I, \tau_{F}=(1,0)$, and condition (8.1) becomes

$$
\Lambda-\Lambda \subset \mathbb{Z} \times \mathbb{R}
$$

Let $\Pi \subset \mathbb{R}$ be the set associated to the spectrum $\Lambda$ defined as in Section 6 , and $\theta(s)$ be the function on $\Pi$ given by Lemma 6.2. By Corollary 7.3, to establish (8.2) it would be enough to show that $\theta(s)$ is constant on $\Pi$.

Let us first consider the case when

$$
\Pi-\Pi \subset \mathbb{Z}
$$

We will show that in this case we must have $\Omega=I \times I$, that is, $\Omega$ is the unit cube, which is not possible as we have assumed that $\Omega$ is not a parallelogram. Indeed, suppose that (8.3) holds, and let $\Lambda^{\prime}$ be the spectrum constructed from $\Lambda$ in Section 7 . Fix a point $\lambda_{0}=\left(t_{0}, s_{0}\right) \in \Lambda^{\prime}$. It follows from Lemma 7.1 and (8.3) that if $\lambda^{\prime}=\left(t^{\prime}, s^{\prime}\right)$ is any point in $\Lambda^{\prime}$ other than $\lambda_{0}$, then at least one of the numbers $t^{\prime}-t_{0}$ and $s^{\prime}-s_{0}$ must be in $\mathbb{Z} \backslash\{0\}$. Now consider the function $f$ defined by (7.4). This function is supported by $\Omega$, and by (17.5) its Fourier transform $\widehat{f}$ vanishes on all the points of $\Lambda^{\prime}$ except from $\lambda_{0}$, since $\widehat{\mathbb{1}}_{I}$ vanishes on $\mathbb{Z} \backslash\{0\}$. Hence $f$ is orthogonal in $L^{2}(\Omega)$ to all the exponentials $\left\{e_{\lambda}\right\}, \lambda \in \Lambda^{\prime} \backslash\left\{\lambda_{0}\right\}$. Since the system $E\left(\Lambda^{\prime}\right)$ is orthogonal and complete in $L^{2}(\Omega)$, this implies that $f$ must coincide a.e. on $\Omega$ with a constant (non-zero) multiple of $e_{\lambda_{0}}$. In particular, $f$ cannot vanish on any subset of $\Omega$ of positive measure. On the other hand, by the definition of $f$ it does vanish on $\Omega \backslash(I \times I)$. This is possible only if $\Omega=I \times I$.

We thus conclude that (8.3) is not possible, so we must have

$$
\Pi-\Pi \not \subset \mathbb{Z} \text {. }
$$

Let us then show that $\theta(s)$ is a constant function on $\Pi$. Indeed, due to (8.4) there exist $s^{\prime}, s^{\prime \prime} \in \Pi$ such that $s^{\prime \prime}-s^{\prime} \notin \mathbb{Z}$. Since $\left\{\widehat{\mathbb{1}}_{I}=0\right\}=\mathbb{Z} \backslash\{0\}$, Corollary 6.3 implies that $\theta\left(s^{\prime}\right)=\theta\left(s^{\prime \prime}\right)$. Observe that for any $s \in \Pi$ we must have $s-s^{\prime} \notin \mathbb{Z}$ or $s-s^{\prime \prime} \notin \mathbb{Z}$, and in either case we obtain, again by Corollary 6.3, that $\theta(s)=\theta\left(s^{\prime}\right)=\theta\left(s^{\prime \prime}\right)$. This shows that $\theta(s)$ must be a constant function on $\Pi$. Lemma 8.2 is therefore proved.

\section{PRISMS AND CYLINDRIC SETS}

9.1. The proof presented in Section 8 that a spectral convex polygon in the plane $\mathbb{R}^{2}$ can tile by translations, eventually relied on showing that the function $\theta(s)$ is constant on the set $\Pi$. In order to show this we had to exclude the case when $\Omega$ is a parallelogram, but since a parallelogram automatically tiles by translations, this loss of generality was innocuous in the proof.

In dimension $d=3$, however, the situation is more complicated. Even if we exclude the case when $\Omega$ is a parallelepiped, one still cannot expect to be able to prove that $\theta(s)$ is a constant function on $\Pi$. Indeed, we have seen in Example 6.5 above that if $\Omega$ is a prism whose base is a spectral set, then the function $\theta(s)$ may attain countably many, arbitrary distinct values. Hence, the role of the parallelogram in dimension $d=2$ will be played not by the parallelepiped, but by the prism, in dimension $d=3$. 
We remind the reader that by a prism in $\mathbb{R}^{d}$ one means a polytope $\Omega$ which can be expressed as the Minkowski sum of a $(d-1)$-dimensional polytope and a line segment.

Notice, however, that while a parallelogram in $\mathbb{R}^{2}$ automatically tiles by translations, this is not so for a prism in $\mathbb{R}^{3}$. Hence it is yet required to prove - necessarily by a different method - that a spectral convex prism in $\mathbb{R}^{3}$ can tile by translations.

Let us formulate this result explicitly:

Theorem 9.1. Let $\Omega$ be a convex prism in $\mathbb{R}^{3}$. If $\Omega$ is spectral, then it tiles by translations.

9.2. A bounded, measurable set $\Omega \subset \mathbb{R}^{d}(d \geqslant 2)$ will be called a cylindric set if it has the form $\Omega=I \times \Sigma$, where $I$ is an interval in $\mathbb{R}$, and $\Sigma$ is a measurable set in $\mathbb{R}^{d-1}$. In this case, the set $\Sigma$ will be called the base of the cylindric set $\Omega$.

If the base $\Sigma$ is a convex polytope in $\mathbb{R}^{d-1}$, then the set $\Omega=I \times \Sigma$ is a convex prism. Conversely, any convex prism in $\mathbb{R}^{d}$ is the affine image of some set of the form $I \times \Sigma$, where $I$ is an interval and $\Sigma$ is a convex polytope in $\mathbb{R}^{d-1}$.

We will deduce Theorem 9.1 from the following result, proved in our paper GL16. The result is valid in all dimensions $d \geqslant 2$ (not just $d=3$ ).

Theorem 9.2 (GL16]). A cylindric set $\Omega=I \times \Sigma$ is spectral (as a set in $\mathbb{R}^{d}$ ) if and only if its base $\Sigma$ is a spectral set (as a set in $\mathbb{R}^{d-1}$ ).

This result thus provides a characterization of the cylindric spectral sets $\Omega$ in terms of the spectrality of their base $\Sigma$.

The "if" part of Theorem 9.2 is obvious. Suppose for simplicity that $I=\left[-\frac{1}{2}, \frac{1}{2}\right]$. If $\Gamma \subset \mathbb{R}^{d-1}$ is a spectrum for $\Sigma$, then it is easy to check that $\mathbb{Z} \times \Gamma$ is a spectrum for $\Omega$, hence $\Omega$ is spectral.

On the other hand, the converse, "only if" part of the theorem (which is what we shall need for our present goal), is non-trivial. Roughly speaking, the difficulty lies in that knowing $\Omega$ to have a spectrum $\Lambda$ in no way implies that $\Lambda$ has a product structure as $\mathbb{Z} \times \Gamma$. In particular, we do not have any obvious candidate for a set $\Gamma \subset \mathbb{R}^{d-1}$ that might serve as a spectrum for $\Sigma$.

Remark. In [GL16] we also gave a similar characterization of the cylindric sets $\Omega$ in $\mathbb{R}^{d}$ which can tile the space by translations. Namely, it was proved there that a cylindric set $\Omega=I \times \Sigma$ tiles if and only if its base $\Sigma$ tiles.

9.3. Theorem 9.1 can now be obtained by a combination of Theorem 9.2 and the result from [IKT03] that a spectral convex polygon in $\mathbb{R}^{2}$ can tile by translations, namely, Theorem 8.1 (for which we have provided an independent proof in Section 8).

Proof of Theorem 9.1. By applying an affine transformation we can assume that $\Omega=$ $I \times \Sigma$, where $I$ is the interval $\left[-\frac{1}{2}, \frac{1}{2}\right]$ and $\Sigma$ is a convex polygon in $\mathbb{R}^{2}$. Since $\Omega$ is spectral, it follows by Theorem 9.2 that also $\Sigma$ is spectral. Hence by Theorem $8.1, \Sigma$ tiles by translations, so there is a set $\Gamma \subset \mathbb{R}^{2}$ such that $\Sigma+\Gamma$ is a tiling of $\mathbb{R}^{2}$. It is then clear that $\Omega$ tiles $\mathbb{R}^{3}$ with the translation set $\mathbb{Z} \times \Gamma$, and this completes the proof. 


\section{PRISMS AND ZONOTOPES}

In Section 9 we explained why the case when the convex polytope $\Omega \subset \mathbb{R}^{3}$ is a prism requires a special treatment in our approach. In this case we obtained a complete solution to our problem, namely, it was proved that if a convex prism in $\mathbb{R}^{3}$ is a spectral set, then it tiles by translations (Theorem 9.1). Hence, in what follows we will be mainly interested in the case when $\Omega$ is not a prism. The goal of the present section is to point out some geometric properties of such an $\Omega$, that will be useful in the analysis of the spectrum later on.

10.1. Let $\Omega \subset \mathbb{R}^{3}$ be a convex polytope, centrally symmetric and with centrally symmetric facets. Let $F$ be a facet of $\Omega$, and $F^{\prime}$ be the opposite facet. Recall that by the central symmetry, $F^{\prime}$ is a translate of $F$, and that we have denoted by $\tau_{F}$ the translation vector in $\mathbb{R}^{3}$ which carries $F^{\prime}$ onto $F$, that is, $F=F^{\prime}+\tau_{F}$.

Suppose now that $A$ is a subfacet of $F$. Then $A$ is the image under the translation by $\tau_{F}$ of a subfacet $A^{\prime}$ of $F^{\prime}$, that is, $A=A^{\prime}+\tau_{F}$. We denote by $H_{F, A}$ the hyperplane which contains the subfacets $A$ and $A^{\prime}$.

Lemma 10.1. If $\Omega$ is not a prism, then for any facet $F$ of $\Omega$ there is a subfacet $A$ such that $\operatorname{int}(\Omega)$ intersects each one of the two open half-spaces bounded by $H_{F, A}$.

Proof. Let $F$ be a facet of $\Omega$. By applying an affine transformation we may assume that

$$
\Omega=-\Omega, \quad F=\left\{\frac{1}{2}\right\} \times \Sigma, \quad F^{\prime}=\left\{-\frac{1}{2}\right\} \times \Sigma,
$$

where $\Sigma$ is a convex polygon in $\mathbb{R}^{2}$ such that $\Sigma=-\Sigma$. Suppose to the contrary that for any subfacet $A$ of $F, \operatorname{int}(\Omega)$ entirely lies within one of the open half-spaces bounded by $H_{F, A}$. The intersection of the closures of all these half-spaces with the set $I \times \mathbb{R}^{2}$, where $I=\left[-\frac{1}{2}, \frac{1}{2}\right]$, is equal to $I \times \Sigma$. Hence $\Omega$ is contained in $I \times \Sigma$. But $\Omega$ also contains $I \times \Sigma$, since $I \times \Sigma$ is the convex hull of the facets $F$ and $F^{\prime}$. We conclude that $\Omega=I \times \Sigma$, which is not possible unless $\Omega$ is a prism. This contradiction ends the proof.

10.2. By a zonotope in $\mathbb{R}^{d}$ one means a polytope which can be represented as the Minkowski sum of a finite number of line segments. A zonotope is a convex, centrally symmetric polytope, and all its facets are also zonotopes. In particular, all the facets of a zonotope are also centrally symmetric.

It is known, see e.g. Sch93, Theorem 3.5.1], that in dimension $d=3$, a convex polytope which has centrally symmetric facets must be a zonotope.

Remark, by the way, that this is not true in dimensions $d \geqslant 4$. A well-known example is the 24 -cell in $\mathbb{R}^{4}$, a convex polytope which tiles by translations, and hence it is centrally symmetric and has centrally symmetric facets, but which is not a zonotope.

10.3. Let again $\Omega \subset \mathbb{R}^{3}$ be a convex polytope, centrally symmetric and with centrally symmetric facets (and hence a zonotope). Let $F$ be a facet of $\Omega$, and $A, B$ be two parallel subfacets of $F$. Let $F^{\prime}$ and $A^{\prime}, B^{\prime}$ be the facet and its two subfacets which are carried onto $F$ and $A, B$ respectively by the translation vector $\tau_{F}$. We denote by $S_{F, A, B}$ the closed slab which lies between the two parallel hyperplanes $H_{F, A}$ and $H_{F, B}$.

Lemma 10.2. Assume that the intersection of $\Omega$ and $S_{F, A, B}$ coincides with the convex hull of the facets $F$ and $F^{\prime}$. Then $\Omega$ is a prism. 
Proof. By applying an affine transformation we may assume that

$$
\Omega=-\Omega, \quad F \subset\left\{x_{1}=\frac{1}{2}\right\}
$$

$F$ is symmetric about the point $\left(\frac{1}{2}, 0,0\right)$,

$$
A=\left\{\frac{1}{2}\right\} \times\left\{\frac{1}{2}\right\} \times I, \quad B=\left\{\frac{1}{2}\right\} \times\left\{-\frac{1}{2}\right\} \times I,
$$

where $I$ denotes as usual the interval $\left[-\frac{1}{2}, \frac{1}{2}\right]$. Hence $F=\left\{\frac{1}{2}\right\} \times \Sigma$, where $\Sigma$ is a convex polygon in $\mathbb{R}^{2}$ such that $\Sigma=-\Sigma$, and such that $\left\{\frac{1}{2}\right\} \times I,\left\{-\frac{1}{2}\right\} \times I$ are facets of $\Sigma$.

The assumption in the lemma thus means that

$$
\Omega \cap(\mathbb{R} \times I \times \mathbb{R})=I \times \Sigma .
$$

Since $\Omega$ is a zonotope, it can be represented as the Minkowski sum of several line segments $S_{1}, S_{2}, \ldots, S_{n}$. Thus we have $\Omega=S_{1}+S_{2}+\cdots+S_{n}$. As $\Omega$ is symmetric about the origin, we can assume that the same is true for each line segment $S_{j}$, that is, $S_{j}=-S_{j}$. We can also assume that no two of the segments $S_{j}$ are parallel.

Now we consider two distinct cases separately. Let us first consider the case when $\Sigma$ is not the cube $I \times I$. In this case there must exist at least one vertex $v$ of $\Sigma$, which belongs to $\operatorname{int}(I \times \mathbb{R})$. Hence $I \times\{v\}$ is a subfacet of $I \times \Sigma$. By (10.1) it follows that $I \times\{v\}$ is also a subfacet of $\Omega$. Each subfacet of $\Omega$ is a translate of one of the $S_{j}$ 's (see, for example, McM71]). Hence one of the line segments, say $S_{1}$, must be equal to $I \times\{0\} \times\{0\}$. It then follows that all the other line segments $S_{2}, \ldots, S_{n}$ must lie in $\{0\} \times \mathbb{R} \times \mathbb{R}$. Indeed, if this is not true for some $S_{j}$, then $S_{1}+S_{j}$ is not contained in $I \times \mathbb{R} \times \mathbb{R}$. But $S_{1}+S_{j}$ is contained in $\Omega$, and $\Omega$ is contained in $I \times \mathbb{R} \times \mathbb{R}$, so this is not possible. Hence all the segments $S_{2}, \ldots, S_{n}$ lie in $\{0\} \times \mathbb{R} \times \mathbb{R}$. It follows that $S_{2}+\cdots+S_{n}=\{0\} \times \Sigma$, and $\Omega=I \times \Sigma$. This shows that $\Omega$ must be a prism.

Now we consider the remaining case, namely, when $\Sigma=I \times I$. In this case, the assumption (10.1) becomes

$$
\Omega \cap(\mathbb{R} \times I \times \mathbb{R})=I \times I \times I .
$$

Hence $\mathbb{R} \times \mathbb{R} \times\left\{\frac{1}{2}\right\}$ and $\mathbb{R} \times \mathbb{R} \times\left\{-\frac{1}{2}\right\}$ are supporting hyperplanes of $\Omega$, and thus $\Omega \subset \mathbb{R} \times \mathbb{R} \times I$. Since $A=\left\{\frac{1}{2}\right\} \times\left\{\frac{1}{2}\right\} \times I$ is a subfacet of $\Omega$, then, as before, one of the line segments, say again $S_{1}$, must be equal to $\{0\} \times\{0\} \times I$. It then follows that all the other line segments $S_{2}, \ldots, S_{n}$ must lie in $\mathbb{R} \times \mathbb{R} \times\{0\}$, since if not, then as before this would contradict the fact that $\Omega \subset \mathbb{R} \times \mathbb{R} \times I$. Hence $S_{2}+\cdots+S_{n}=P \times\{0\}$ for a certain convex polygon $P \subset \mathbb{R}^{2}$, and $\Omega=P \times I$. Again we obtain that $\Omega$ must be a prism, so this proves the lemma.

\section{Structure of Spectrum, III}

In this section our goal is to relate the geometric observations made in Section 10 to the spectrality problem for convex polytopes in dimension $d=3$. More specifically, we will see how one can use the assumption that $\Omega$ is not a prism, in order to obtain new information on the structure of the spectrum $\Lambda$. 
11.1. Let $\Omega \subset \mathbb{R}^{3}$ be a convex polytope, centrally symmetric and with centrally symmetric facets. Assume, as before, that $\Omega=-\Omega$, that is, $\Omega$ is symmetric about the origin, $F$ is a facet of $\Omega$ contained in $\left\{x_{1}=\frac{1}{2}\right\}$, and that the center of $F$ is the point $\left(\frac{1}{2}, 0,0\right)$. Hence $F=\left\{\frac{1}{2}\right\} \times \Sigma$, where $\Sigma$ is a convex polygon in $\mathbb{R}^{2}$ such that $\Sigma=-\Sigma$.

Suppose also that $\Lambda$ is a spectrum for $\Omega$. Let $\Pi \subset \mathbb{R}^{2}$ be the set associated to the spectrum $\Lambda$ defined as in Section 6, and $\theta(s)$ be the function on $\Pi$ given by Lemma 6.2. We also let $\Lambda^{\prime}$ be the new spectrum constructed from $\Lambda$ in Section 7 .

Recall that to each point $\left(t_{0}, s_{0}\right) \in \mathbb{R} \times \mathbb{R}^{2}$ we have associated a function $f$, supported by $\Omega$, defined by (17.4). As an element of $L^{2}(\Omega)$ this function $f$ admits a Fourier expansion with respect to the spectrum $\Lambda^{\prime}$, given by

$$
f=\frac{1}{|\Omega|} \sum_{\lambda \in \Lambda^{\prime}} \widehat{f}(\lambda) e_{\lambda} .
$$

By Lemma 2.1 the series on the right hand side of (11.1) converges in $L^{2}$ on any bounded set to a measurable function $\tilde{f}$ on $\mathbb{R}^{3}$, and $f$ coincides with $\tilde{f}$ a.e. on $\Omega$.

We now observe that for certain values of $\left(t_{0}, s_{0}\right)$, the Fourier expansion of $f$ with respect to the spectrum $\Lambda^{\prime}$ consists of exceptionally few terms:

Lemma 11.1. Let $\left(t_{0}, s_{0}\right)$ be a point belonging to $\left(\mathbb{Z}+\theta_{j}\right) \times \Pi_{j}$ for some $j$, and let $f$ be the function defined by (7.4). Then the Fourier expansion (11.1) of $f$ with respect to the spectrum $\Lambda^{\prime}$ consists only of terms corresponding to $\lambda \in \Lambda^{\prime} \cap\left(\left\{t_{0}\right\} \times \Pi_{j}\right)$.

In other words, all the coefficients $\widehat{f}(\lambda)$ in the expansion (11.1) must vanish except for possibly those which correspond to $\lambda=(t, s) \in \Lambda^{\prime}$ such that $t=t_{0}$ and $s \in \Pi_{j}$.

Proof of Lemma 11.1. If $(t, s) \in \Lambda^{\prime}$, then by Lemma 7.1 there is $k$ such that $t \in \mathbb{Z}+\theta_{k}$ and $s \in \Pi_{k}$. If $k \neq j$ then $\widehat{\mathbb{1}}_{\Sigma}\left(s-s_{0}\right)=0$ due to (7.3), and it follows from (7.5) that $\widehat{f}(t, s)=0$. If $k=j$ then both $t_{0}$ and $t$ belong to $\mathbb{Z}+\theta_{j}$, hence $t-t_{0}$ is an integer. Since $\widehat{\mathbb{1}}_{I}$ vanishes on $\mathbb{Z} \backslash\{0\}$, it follows again by (7.5) that $\widehat{f}(t, s)=0$ unless $t=t_{0}$. This shows that in the series (11.1) the non-zero coefficients can only correspond to $\lambda=(t, s)$ such that $t=t_{0}$ and $s \in \Pi_{j}$. This proves the claim.

Remark. It may be interesting to notice that Lemma 11.1 implies that $\Lambda^{\prime}$ must contain points from each one of the sets $\left\{t_{0}\right\} \times \Pi_{j}$, where $t_{0}$ goes through the elements of $\mathbb{Z}+\theta_{j}$.

11.2. Now suppose that $\Omega$ is not a prism. Then by Lemma 10.1 there is a subfacet $A$ of $F$ such that $\operatorname{int}(\Omega)$ intersects each one of the two open half-spaces bounded by the hyperplane $H_{F, A}$. Let us assume, for simplicity, that this subfacet is $A=\left\{\frac{1}{2}\right\} \times\left\{\frac{1}{2}\right\} \times I$, where $I=\left[-\frac{1}{2}, \frac{1}{2}\right]$ (later on, the general situation will be reduced to this case by applying an affine transformation). Thus $\left\{\frac{1}{2}\right\} \times I$ is a facet of the convex polygon $\Sigma$.

We can now use Lemma 11.1 to obtain some additional information on the structure of the components $\Pi_{j}$ of the set $\Pi$.

Lemma 11.2. For each $j$ we have

$$
\Pi_{j}-\Pi_{j} \not \subset \mathbb{Z} \times \mathbb{R} .
$$

Proof. Suppose that (11.2) is not true for some $j$. By translating the spectrum $\Lambda$ we can assume that $\Pi_{j}$ contains the origin, and hence

$$
\Pi_{j} \subset \mathbb{Z} \times \mathbb{R} .
$$


Choose a point $\left(t_{0}, s_{0}\right) \in\left(\mathbb{Z}+\theta_{j}\right) \times \Pi_{j}$, and let $f$ be the function associated to this point defined by (7.4). By Lemma 11.1 and due to (11.3), the Fourier expansion of $f$ with respect to $\Lambda^{\prime}$ consists only of exponentials $e_{\lambda}$ such that $\lambda \in \Lambda^{\prime} \cap(\mathbb{R} \times \mathbb{Z} \times \mathbb{R})$. It follows (Lemma 2.1) that the right-hand side of (11.1) is a function $\tilde{f}$ on $\mathbb{R}^{3}$ which is periodic with respect to the vector $(0,1,0)$, and $f$ coincides with $\tilde{f}$ a.e. on $\Omega$.

Recall that we have chosen the subfacet $A$ of $F$ (using Lemma 10.1) such that int $(\Omega$ ) intersects each one of the two open half-spaces bounded by the hyperplane $H_{F, A}$. Since it was assumed that $A=\left\{\frac{1}{2}\right\} \times\left\{\frac{1}{2}\right\} \times I$, this means that $H_{F, A}=\left\{x_{2}=\frac{1}{2}\right\}$, and hence

$$
\Omega \not \subset\left\{x_{2} \leqslant \frac{1}{2}\right\} .
$$

Recall also that $F=\left\{\frac{1}{2}\right\} \times \Sigma$, where $\Sigma$ is a convex polygon in $\mathbb{R}^{2}, \Sigma=-\Sigma$, and $\left\{\frac{1}{2}\right\} \times I$ is a face of $\Sigma$. By convexity, $\Sigma$ contains the unit square $I \times I$, and hence $I \times \Sigma$ contains the unit cube $I \times I \times I$. Thus $|\tilde{f}|=|f|=1$ a.e. on $I \times I \times I$. By the periodicity of $\tilde{f}$ this implies that $|\tilde{f}|=1$ a.e. on $I \times \mathbb{R} \times I$. In particular, $|\tilde{f}|=1$ a.e. on the set

$$
\Omega \cap(I \times(\mathbb{R} \backslash I) \times I) .
$$

On the other hand, the set (11.5) is disjoint from $I \times \Sigma$, hence $|f|=0$ on this set. It follows that the set (11.5) cannot have positive measure, and therefore

$$
\Omega \cap(I \times \mathbb{R} \times I)=I \times I \times I .
$$

This implies that $\left\{x_{2}=\frac{1}{2}\right\}$ is a supporting hyperplane of $\Omega$, which contradicts (11.4).

Lemma 11.3. For each $j$ we have

$$
\Pi_{j}-\Pi_{j} \not \subset \mathbb{R} \times \mathbb{Z}
$$

Proof. We argue in a way similar to the proof of the previous lemma. If (11.6) is violated for some $j$, then by translating $\Lambda$ we can assume that

$$
\Pi_{j} \subset \mathbb{R} \times \mathbb{Z}
$$

Hence, choosing a point $\left(t_{0}, s_{0}\right) \in\left(\mathbb{Z}+\theta_{j}\right) \times \Pi_{j}$, the corresponding function $f$ defined by (7.4) coincides a.e. on $\Omega$ with a function $\tilde{f}$ on $\mathbb{R}^{3}$, which by (11.7) and Lemma 11.1 is periodic with respect to the vector $(0,0,1)$.

Since we have $|\tilde{f}|=|f|=1$ a.e. on $I \times I \times I$, the periodicity of $\tilde{f}$ implies that $|\tilde{f}|=1$ a.e. on $I \times I \times \mathbb{R}$. In particular, $|\tilde{f}|=1$ a.e. on the set

$$
\Omega \cap(I \times((I \times \mathbb{R}) \backslash \Sigma)) .
$$

But since this set is disjoint from $I \times \Sigma$, we have $|f|=0$ on the set (11.8). So the set (11.8) cannot have positive measure, and therefore

$$
\Omega \cap(I \times I \times \mathbb{R})=I \times \Sigma .
$$

By Lemma 10.2 this is possible only if $\Omega$ is a prism, so this concludes the proof.

Lemma 11.4. Let $X$ be a subset of an abelian group $G$, and let $H_{1}$ and $H_{2}$ be two subgroups of $G$. Assume that

$$
X-X \subset H_{1} \cup H_{2} .
$$

Then $X-X \subset H_{1}$ or $X-X \subset H_{2}$. 
Proof. Suppose that $X-X \not \subset H_{1}$, so there exist $x, y \in X$ such that $x-y \notin H_{1}$. Then by (11.9) we have $x-y \in H_{2}$. The property $x-y \notin H_{1}$ implies that for each $z \in X$ we must have $z-x \notin H_{1}$ or $z-y \notin H_{1}$. But in either case, it follows from (11.9) that $z \in x+H_{2}=y+H_{2}$, so we conclude that $X \subset x+H_{2}=y+H_{2}$. Thus $X-X \subset H_{2}$.

Corollary 11.5. For each $j$ we have

$$
\Pi_{j}-\Pi_{j} \not \subset(\mathbb{Z} \times \mathbb{R}) \cup(\mathbb{R} \times \mathbb{Z}) .
$$

This is an immediate consequence of Lemmas 11.2, 11.3 and 11.4.

\section{Structure of spectrum, IV}

In this section we continue to analyze the structure of the spectrum of a convex polytope $\Omega$ in dimension $d=3$. Although we are mainly interested in the case when $\Omega$ is not a prism, we will not need to assume this in the present section.

12.1. Let $\Omega$ be a convex polytope in $\mathbb{R}^{3}$, centrally symmetric and with centrally symmetric facets. Assume that $\Omega$ is in our "standard position", namely, $\Omega=-\Omega, F$ is a facet of $\Omega$ contained in $\left\{x_{1}=\frac{1}{2}\right\}$, and $F$ is symmetric about the point $\left(\frac{1}{2}, 0,0\right)$. Assume also that $A=\left\{\frac{1}{2}\right\} \times\left\{\frac{1}{2}\right\} \times I$ is a subfacet of $F$, where $I=\left[-\frac{1}{2}, \frac{1}{2}\right]$. Hence $F=\left\{\frac{1}{2}\right\} \times \Sigma$, where $\Sigma$ is a convex polygon in $\mathbb{R}^{2}, \Sigma=-\Sigma$, and $\left\{\frac{1}{2}\right\} \times I$ is a facet of $\Sigma$.

Suppose that $\Lambda$ is a spectrum for $\Omega$. Let $\Pi \subset \mathbb{R}^{2}$ be the set associated to the spectrum $\Lambda$ defined in Section 6, and $\theta(s)$ be the function on $\Pi$ given by Lemma 6.2. Recall that in Section 7, a new spectrum $\Lambda^{\prime}$ was constructed from the given spectrum $\Lambda$, by taking the weak limit of a sequence of translates of $\Lambda$. The new spectrum $\Lambda^{\prime}$ was shown (Lemma 7.1) to enjoy a particular structure, namely

$$
\Lambda^{\prime} \subset \bigcup_{j \geqslant 0}\left(\mathbb{Z}+\theta_{j}\right) \times \Pi_{j}
$$

where $\Pi_{j}$ are the components of the set $\Pi$, and $\theta_{j}$ are respectively the values of the function $\theta(s)$ on these components. The sets $\Pi_{j}$ were shown (Corollary 6.3) to satisfy

$$
\Pi_{k}-\Pi_{j} \subset\left\{\widehat{\mathbb{1}}_{\Sigma}=0\right\} \quad(j \neq k) .
$$

When we want to further analyze the structure of the spectrum in dimension $d=3$, a new complication arises, that was not present in the case $d=2$. Namely, the zero set $\left\{\widehat{\mathbb{1}}_{\Sigma}=0\right\}$ is not known explicitly, except in the special case when $\Sigma$ is the cube $I \times I$. In order to address this difficulty, a further limiting procedure will now be performed on the spectrum $\Lambda^{\prime}$, yielding a third spectrum $\Lambda^{\prime \prime}$ of $\Omega$.

12.2. The new spectrum $\Lambda^{\prime \prime}$ is constructed as follows. Consider the sequence of translates of the spectrum $\Lambda^{\prime}$ given by

$$
\Lambda^{\prime}-r \cdot(0,1,0), \quad r=1,2,3, \ldots
$$

As in Section 7 we may extract from this sequence a subsequence

$$
\Lambda^{\prime}-r_{n} \cdot(0,1,0), \quad r_{n} \rightarrow \infty,
$$

which converges weakly to some set $\Lambda^{\prime \prime}$, which is again a spectrum of $\Omega$.

According to (12.1) we may form a partition of the spectrum $\Lambda^{\prime}$ into sets defined by

$$
\Lambda_{j}^{\prime}:=\Lambda^{\prime} \cap\left(\left(\mathbb{Z}+\theta_{j}\right) \times \Pi_{j}\right)
$$


It would be convenient for us to know that for each $j$, the sequence of translates

$$
\Lambda_{j}^{\prime}-r_{n} \cdot(0,1,0)
$$

of each component $\Lambda_{j}^{\prime}$ has a weak limit as $n \rightarrow \infty$. This does not follow automatically from the weak convergence of the sequence (12.3), though, since we have not excluded the possibility that there may be infinitely many $\theta_{j}$ 's and that they may have accumulation points. Nevertheless, we can assume that (12.5) has a weak limit as $n \rightarrow \infty$ for each $j$, simply by selecting a further subsequence if necessary.

We shall denote by $\Lambda_{j}^{\prime \prime}$ the weak limit of (12.5). Observe that a point $(t, u, v) \in \mathbb{R}^{3}$ belongs to $\Lambda_{j}^{\prime \prime}$ if and only if there is a sequence $\left(t_{n}, u_{n}, v_{n}\right) \in \Lambda_{j}^{\prime}$ such that

$$
\left(t_{n}, u_{n}-r_{n}, v_{n}\right) \rightarrow(t, u, v), \quad n \rightarrow \infty .
$$

Remark that while by Lemma 11.1 none of the components $\Lambda_{j}^{\prime}$ may be empty, this is not true for the sets $\Lambda_{j}^{\prime \prime}$ that we cannot exclude some of which to be empty.

It follows from (12.4) that

$$
\Lambda_{j}^{\prime \prime} \subset \Lambda^{\prime \prime} \cap\left(\left(\mathbb{Z}+\theta_{j}\right) \times \mathbb{R}^{2}\right),
$$

hence the sets $\Lambda_{j}^{\prime \prime}$ are disjoint subsets of $\Lambda^{\prime \prime}$. Remark, however, that these sets do not necessarily form a partition of $\Lambda^{\prime \prime}$, namely, their union need not be equal to the whole $\Lambda^{\prime \prime}$. Again, this may happen only if there are infinitely many $\theta_{j}$ 's. An example of such a situation can be obtained if $\Omega$ is a prism whose base is a spectral set. Indeed, we have seen in Example 6.5 that in such a case the function $\theta(s)$ may attain countably many, arbitrary distinct values, and that the components $\Pi_{j}$ of the set $\Pi$ may be singletons. This implies that every $\Lambda_{j}^{\prime \prime}$ is empty, while $\Lambda^{\prime \prime}$ certainly cannot be empty being a spectrum for $\Omega$.

This makes it necessary for us in general to consider also the subset of $\Lambda^{\prime \prime}$ defined by

$$
\Lambda_{\infty}^{\prime \prime}:=\Lambda^{\prime \prime} \backslash \bigcup_{j \geqslant 0} \Lambda_{j}^{\prime \prime}
$$

Lemma 12.1. Let $(t, u, v) \in \mathbb{R}^{3}$. Then $(t, u, v)$ belongs to $\Lambda_{\infty}^{\prime \prime}$ if and only if there is a sequence $k_{n} \rightarrow \infty$, and for each $n$ there is a point $\left(t_{n}, u_{n}, v_{n}\right) \in \Lambda_{k_{n}}^{\prime}$, such that

$$
\left(t_{n}, u_{n}-r_{n}, v_{n}\right) \rightarrow(t, u, v), \quad n \rightarrow \infty .
$$

Proof. Suppose first that $(t, u, v)$ is a point in $\Lambda_{\infty}^{\prime \prime}$. Then $(t, u, v) \in \Lambda^{\prime \prime}$, and since $\Lambda^{\prime \prime}$ is the weak limit of (12.3), there exist $\left(t_{n}, u_{n}, v_{n}\right) \in \Lambda^{\prime}$ such that $\left(t_{n}, u_{n}-r_{n}, v_{n}\right) \rightarrow(t, u, v)$. Due to (12.1) and (12.4), for each $n$ there is $k_{n} \geqslant 0$ such that $\left(t_{n}, u_{n}, v_{n}\right) \in \Lambda_{k_{n}}^{\prime}$. If $k_{n} \nrightarrow \rightarrow \infty$, then $k_{n}$ admits infinitely often a certain value, say $k_{n}=j$ for infinitely many $n$ 's. But this implies that $(t, u, v)$ must belong to the weak limit of (12.5), and hence $(t, u, v) \in \Lambda_{j}^{\prime \prime}$, so it cannot lie in $\Lambda_{\infty}^{\prime \prime}$. Hence we must have $k_{n} \rightarrow \infty$.

Conversely, suppose that the point $(t, u, v)$ satisfies the condition in the lemma. The condition implies that $(t, u, v)$ belongs to the weak limit of (12.3), hence $(t, u, v) \in \Lambda^{\prime \prime}$. If $(t, u, v)$ is not in $\Lambda_{\infty}^{\prime \prime}$, then it belongs to one of the sets $\Lambda_{j}^{\prime \prime}$. But then we must have $k_{n}=j$ for all sufficiently large $n$, so $k_{n} \nrightarrow \rightarrow \infty$, a contradiction. Hence $(t, u, v) \in \Lambda_{\infty}^{\prime \prime}$.

We also point out that the inclusion (12.6) is not necessarily an equality, as the right hand side of (12.6) may contain elements of $\Lambda_{\infty}^{\prime \prime}$. 
12.3. Now we establish some properties satisfied by the new spectrum $\Lambda^{\prime \prime}$ and its components $\Lambda_{k}^{\prime \prime}(0 \leqslant k \leqslant \infty)$. The first property is derived from the condition (12.2).

Lemma 12.2. For each $0 \leqslant j, k \leqslant \infty, j \neq k$, we have

$$
\Lambda_{k}^{\prime \prime}-\Lambda_{j}^{\prime \prime} \subset \mathbb{R} \times\left\{\widehat{\mathbb{1}}_{\Sigma}=0\right\} .
$$

Proof. By symmetry we may assume that $0 \leqslant j<k \leqslant \infty$. Let $(t, u, v) \in \Lambda_{j}^{\prime \prime}$ and $\left(t^{\prime}, u^{\prime}, v^{\prime}\right) \in \Lambda_{k}^{\prime \prime}$. Then there exist two sequences

$$
\left(t_{n}, u_{n}, v_{n}\right) \in \Lambda_{j}^{\prime}, \quad\left(t_{n}, u_{n}-r_{n}, v_{n}\right) \rightarrow(t, u, v),
$$

and

$$
\left(t_{n}^{\prime}, u_{n}^{\prime}, v_{n}^{\prime}\right) \in \Lambda_{k_{n}}^{\prime}, \quad\left(t_{n}^{\prime}, u_{n}^{\prime}-r_{n}, v_{n}^{\prime}\right) \rightarrow\left(t^{\prime}, u^{\prime}, v^{\prime}\right),
$$

where $k_{n}=k$ in the case when $k$ is finite, or $k_{n} \rightarrow \infty$ if $k=\infty$ (Lemma 12.1). In any case we have $k_{n} \neq j$ for all sufficiently large $n$. Since by (12.4) we have

$$
\left(u_{n}, v_{n}\right) \in \Pi_{j}, \quad\left(u_{n}^{\prime}, v_{n}^{\prime}\right) \in \Pi_{k_{n}},
$$

then it follows from (12.2) that

$$
\left(t_{n}^{\prime}, u_{n}^{\prime}-r_{n}, v_{n}^{\prime}\right)-\left(t_{n}, u_{n}-r_{n}, v_{n}\right)=\left(t_{n}^{\prime}-t_{n}, u_{n}^{\prime}-u_{n}, v_{n}^{\prime}-v_{n}\right) \in \mathbb{R} \times\left\{\widehat{\mathbb{1}}_{\Sigma}=0\right\} .
$$

Letting $n \rightarrow \infty$ we obtain

$$
\left(t^{\prime}, u^{\prime}, v^{\prime}\right)-(t, u, v) \in \mathbb{R} \times\left\{\widehat{\mathbb{1}}_{\Sigma}=0\right\},
$$

which confirms (12.7).

Lemma 12.2 shows that the structure (12.2) is basically preserved in the new spectrum $\Lambda^{\prime \prime}$ and its components $\Lambda_{k}^{\prime \prime}(0 \leqslant k \leqslant \infty)$. However, our motivation for introducing this new spectrum is due to the following lemma:

Lemma 12.3. Let $0 \leqslant j<\infty, 0 \leqslant k \leqslant \infty, k \neq j$. Then

$$
\Lambda_{k}^{\prime \prime}-\mathbb{R} \times \Pi_{j} \subset(\mathbb{R} \times \mathbb{Z} \times \mathbb{R}) \cup(\mathbb{R} \times \mathbb{R} \times(\mathbb{Z} \backslash\{0\})) .
$$

In other words, if $\left(u_{0}, v_{0}\right) \in \Pi_{j}$ and if $(t, u, v) \in \Lambda_{k}^{\prime \prime}$, then $u-u_{0} \in \mathbb{Z}$ or $v-v_{0} \in \mathbb{Z} \backslash\{0\}$.

This lemma is similar in spirit to Lemma 6.2. To see the resemblance between the two lemmas, recall that $\left\{\frac{1}{2}\right\} \times I$ is a facet of the polygon $\Sigma$, and $\left\{\widehat{\mathbb{1}}_{I}=0\right\}=\mathbb{Z} \backslash\{0\}$. The assertion of (12.8) is equivalent to the statement that if $\left(u_{0}, v_{0}\right) \in \Pi_{j},(t, u, v) \in \Lambda_{k}^{\prime \prime}$, and if $\widehat{\mathbb{1}}_{I}\left(v-v_{0}\right) \neq 0$, then $u \in \mathbb{Z}+u_{0}$. The proof is also similar to that of Lemma 6.2.

Proof of Lemma 12.3. Let $\left(u_{0}, v_{0}\right) \in \Pi_{j}$ and $(t, u, v) \in \Lambda_{k}^{\prime \prime}$. Regardless of whether $k$ is finite or not, there is a sequence $k_{n}$ and there are points $\left(t_{n}, u_{n}, v_{n}\right) \in \Lambda_{k_{n}}^{\prime}$ such that

$$
\left(t_{n}, u_{n}-r_{n}, v_{n}\right) \rightarrow(t, u, v), \quad n \rightarrow \infty .
$$

Indeed, if $0 \leqslant k<\infty$ then $k_{n}=k$ for all $n$, while if $k=\infty$ then $k_{n} \rightarrow \infty$ (Lemma 12.1). In any case, we have $k_{n} \neq j$ for all sufficiently large $n$. Since $\left(t_{n}, u_{n}, v_{n}\right) \in \Lambda_{k_{n}}^{\prime}$ we have $\left(u_{n}, v_{n}\right) \in \Pi_{k_{n}}$ by (12.4). Hence by (12.2) this implies that

$$
\widehat{\mathbb{1}}_{\Sigma}\left(u_{n}-u_{0}, v_{n}-v_{0}\right)=0,
$$

for all sufficiently large $n$.

Observe that since $r_{n} \rightarrow \infty$, (12.9) implies that also $u_{n} \rightarrow \infty$. Hence using Lemma 6.1 for the polygon $\Sigma$ and its facet $\left\{\frac{1}{2}\right\} \times I$, it follows from (12.10) that

$$
\sin \pi\left(u_{n}-u_{0}\right) \cdot \widehat{\mathbb{1}}_{I}\left(v_{n}-v_{0}\right) \rightarrow 0, \quad n \rightarrow \infty .
$$


Indeed, the polygon $\Sigma$ is centrally symmetric and it has centrally symmetric facets, as the facets of $\Sigma$ are line segments, hence all the conditions of Lemma 6.1 are satisfied.

Now suppose that $v-v_{0} \notin \mathbb{Z} \backslash\{0\}$. Then $v-v_{0}$ is not contained in the zero set of $\widehat{\mathbb{1}}_{I}$, and hence $\left|\widehat{\mathbb{1}}_{I}\left(v_{n}-v_{0}\right)\right|$ remains bounded away from zero as $n \rightarrow \infty$. So we must have $\sin \pi\left(u_{n}-u_{0}\right) \rightarrow 0$, or equivalently, $\operatorname{dist}\left(u_{n}-u_{0}, \mathbb{Z}\right) \rightarrow 0$. But since $r_{n}$ is an integer, (12.9) implies that also $\operatorname{dist}\left(u_{n}-u, \mathbb{Z}\right) \rightarrow 0$. It follows that

$$
\operatorname{dist}\left(u-u_{0}, \mathbb{Z}\right) \leqslant \operatorname{dist}\left(u_{n}-u_{0}, \mathbb{Z}\right)+\operatorname{dist}\left(u_{n}-u, \mathbb{Z}\right) \rightarrow 0 .
$$

We conclude that $u-u_{0} \in \mathbb{Z}$ as required.

From the previous lemma it is easy to deduce the next one:

Lemma 12.4. For each $0 \leqslant j, k \leqslant \infty, j \neq k$, we have

$$
\Lambda_{k}^{\prime \prime}-\Lambda_{j}^{\prime \prime} \subset(\mathbb{R} \times \mathbb{Z} \times \mathbb{R}) \cup(\mathbb{R} \times \mathbb{R} \times(\mathbb{Z} \backslash\{0\})) .
$$

Actually we will not use Lemma 12.4 in what follows. We state it merely to demonstrate an essential advantage of the newly constructed spectrum $\Lambda^{\prime \prime}$. On one hand, according to (12.7) it basically inherits the structure of the previously constructed spectrum $\Lambda^{\prime}$, while on the other hand, condition (12.11) reveals an extra structure in $\Lambda^{\prime \prime}$.

Since the proof of Lemma 12.4 is quite short, we include it for completeness.

Proof of Lemma 12.4. By symmetry we may assume that $0 \leqslant j<k \leqslant \infty$. Let $(t, u, v) \in$ $\Lambda_{k}^{\prime \prime}$. Then by Lemma 12.3 the set $\mathbb{R} \times \Pi_{j}$ must be contained in

$$
(\mathbb{R} \times(u+\mathbb{Z}) \times \mathbb{R}) \cup(\mathbb{R} \times \mathbb{R} \times(v+(\mathbb{Z} \backslash\{0\}))) .
$$

Due to (12.4) we have $\Lambda_{j}^{\prime} \subset \mathbb{R} \times \Pi_{j}$, hence also the set $\Lambda_{j}^{\prime}$ is contained in (12.12). Since the set (12.12) is invariant under translations by vectors in $\{0\} \times \mathbb{Z} \times\{0\}$, it follows that all the sets (12.5) are also contained in (12.12), and hence the same is true for their weak limit $\Lambda_{j}^{\prime \prime}$. This implies that $\Lambda_{j}^{\prime \prime}-(t, u, v)$ is contained in the set on the right-hand side of (12.11). As $(t, u, v)$ was an arbitrary element of $\Lambda_{k}^{\prime \prime}$, this establishes (12.11).

\section{Auxiliary lemmas}

In this section we establish some specific facts about the spectrum of a convex polytope $\Omega$ that will be used later on. These facts are true in arbitrary dimension, so in the present section we do not restrict the discussion to three dimensions.

13.1. Let $\Omega \subset \mathbb{R}^{d}$ be a convex polytope. Let $F$ and $F^{\prime}$ be two parallel facets of $\Omega$, and assume that $F \subset\left\{x_{1}=\frac{1}{2}\right\}, F^{\prime} \subset\left\{x_{1}=-\frac{1}{2}\right\}$, and that $F$ is the image of $F^{\prime}$ under translation by the vector $\vec{e}_{1}$. These assumptions imply that

$$
F=\left\{\frac{1}{2}\right\} \times \Sigma, \quad F^{\prime}=\left\{-\frac{1}{2}\right\} \times \Sigma,
$$

where $\Sigma$ is a convex polytope in $\mathbb{R}^{d-1}$.

Assume also that $\Omega$ is spectral, and let $\Lambda$ be a spectrum for $\Omega$.

Lemma 13.1. If $\Omega$ is not a prism, then $\Lambda$ cannot contain any set of the form

$$
(\mathbb{Z}+\theta) \times\{s\},
$$

where $\theta \in \mathbb{R}$ and $s \in \mathbb{R}^{d-1}$. 
Proof. Suppose to the contrary that $\Lambda$ does contain a set of the form (13.1). This implies that the set $\Lambda-\Lambda$ contains $\mathbb{Z} \times\{0\}$. On the other hand, since $\Lambda$ is a spectrum for $\Omega$, the set $\Lambda-\Lambda$ must be contained in $\left\{\widehat{\mathbb{1}}_{\Omega}=0\right\} \cup\{0\}$. We conclude that

$$
\widehat{\mathbb{1}}_{\Omega}(k, 0)=0, \quad k \in \mathbb{Z} \backslash\{0\} .
$$

For each $x \in \mathbb{R}$ denote by $\Omega_{x}$ the $(d-1)$-dimensional polytope obtained by the intersection of $\Omega$ with the hyperplane $\{x\} \times \mathbb{R}^{d-1}$, and let $\varphi(x)$ be the $(d-1)$-dimensional volume of $\Omega_{x}$. Then the function $\varphi$ vanishes off the interval $I=\left[-\frac{1}{2}, \frac{1}{2}\right]$, it is continuous on $I$, and $\varphi\left(\frac{1}{2}\right)=\varphi\left(-\frac{1}{2}\right)=|\Sigma|$. Notice that, by convexity, $\Omega_{x}$ contains $\{x\} \times \Sigma$ for every $x \in I$. In particular this implies that $\varphi(x) \geqslant|\Sigma|, x \in I$.

It follows from the definition of the function $\varphi$ that its Fourier transform is given by

$$
\widehat{\varphi}(t)=\widehat{\mathbb{1}}_{\Omega}(t, 0), \quad t \in \mathbb{R} .
$$

Combining this with (13.2) we obtain that $\widehat{\varphi}$ vanishes on $\mathbb{Z} \backslash\{0\}$. Since $\varphi$ is supported on $I$, this implies that $\varphi$ is orthogonal in $L^{2}(I)$ to all the exponentials $\left\{e_{k}\right\}, k \in \mathbb{Z} \backslash\{0\}$. But as the system $E(\mathbb{Z})$ is orthogonal and complete in $L^{2}(I)$, this is possible only if $\varphi$ is constant on $I$. Hence $\varphi(x)=|\Sigma|$ for all $x \in I$. In turn, this implies that $\Omega_{x}=\{x\} \times \Sigma$, $x \in I$. We conclude that $\Omega=I \times \Sigma$, and so $\Omega$ is a prism, a contradiction.

Remark. One can see from the proof that the only property of the set (13.1) that was actually used was that its difference set contains $\mathbb{Z} \times\{0\}$. Hence the lemma remains true if (13.1) is replaced by any other set for which the latter property is satisfied.

13.2. Denote by $Q=I^{d-1}$ the unit cube in $\mathbb{R}^{d-1}$. As usual, $I$ is the interval $\left[-\frac{1}{2}, \frac{1}{2}\right]$.

Lemma 13.2. Assume that $\Sigma$ contains $Q$. If $\Omega$ is not a prism, then $\Lambda$ cannot be covered by the union of two translates of $\mathbb{Z}^{d}$.

Proof. Suppose to the contrary that $\Lambda$ is contained in the union of two translates of $\mathbb{Z}^{d}$. By translating $\Lambda$ we may assume that

$$
\Lambda \subset \mathbb{Z}^{d} \cup\left(\mathbb{Z}^{d}+\tau\right)
$$

for some $\tau \in \mathbb{R}^{d}$. According to Lemma 13.1, the spectrum $\Lambda$ cannot contain the whole set $\mathbb{Z} \times\{0\}$. This implies that by further translating $\Lambda$ by a certain vector in $\mathbb{Z} \times\{0\}$, we may additionally assume that $\Lambda$ does not contain the origin.

Since $\Sigma$ is assumed to contain $Q$, and since by convexity $\Omega$ contains $I \times \Sigma$, then $\Omega$ must contain $I \times Q$, the unit cube in $\mathbb{R}^{d}$. Hence the function $f=\mathbb{1}_{I \times Q}$ is supported by $\Omega$. Consider the Fourier expansion (2.3) of this function $f$. Since $\widehat{f}$ vanishes on all the points of $\mathbb{Z}^{d}$ except the origin, and since the origin does not belong to $\Lambda$, it follows from (13.3) that only exponentials $e_{\lambda}$ such that $\lambda \in \Lambda \cap\left(\mathbb{Z}^{d}+\tau\right)$ may have a non-zero coefficient in the expansion (2.3). Hence by Lemma 2.1 the right-hand side of (2.3) represents a function $\tilde{f}$ of the form

$$
\tilde{f}(x)=e^{2 \pi i\langle\tau, x\rangle} g(x), \quad x \in \mathbb{R}^{d},
$$

where $g$ is some $\mathbb{Z}^{d}$-periodic function, and $f$ coincides with $\tilde{f}$ a.e. on $\Omega$. Notice that $|g|=|\tilde{f}|=|f|=1$ a.e. on $I \times Q$. By the periodicity of $g$ this implies that $|g|=1$ a.e. on $\mathbb{R}^{d}$. Hence $|f|=|\tilde{f}|=|g|=1$ a.e. on $\Omega$. In particular, $f$ cannot vanish on any subset of $\Omega$ of positive measure. On the other hand, by the definition of $f$ it does vanish on $\Omega \backslash(I \times Q)$. This is possible only if $\Omega=I \times Q$, namely, $\Omega$ is the unit cube in $\mathbb{R}^{d}$. But this contradicts the assumption that $\Omega$ is not a prism, so the proof is complete. 


\section{Structure of spectrum, V}

In this section we complete the analysis of the spectrum in dimension $d=3$.

14.1. Our assumptions will be the following.

Let $\Omega \subset \mathbb{R}^{3}$ be a convex polytope, centrally symmetric and with centrally symmetric facets. We assume that $\Omega$ is not a prism. Suppose that $\Omega$ is in the "standard position", namely, $\Omega=-\Omega, F$ is a facet of $\Omega$ contained in $\left\{x_{1}=\frac{1}{2}\right\}$, and $F$ is symmetric about the point $\left(\frac{1}{2}, 0,0\right)$. Hence $F=\left\{\frac{1}{2}\right\} \times \Sigma$, where $\Sigma$ is a convex polygon in $\mathbb{R}^{2}$ such that $\Sigma=-\Sigma$. We assume that $A=\left\{\frac{1}{2}\right\} \times\left\{\frac{1}{2}\right\} \times I$ is a subfacet of $F$, where $I=\left[-\frac{1}{2}, \frac{1}{2}\right]$, and therefore $\left\{\frac{1}{2}\right\} \times I$ is a facet of $\Sigma$. We also suppose that $\operatorname{int}(\Omega)$ intersects each one of the two open half-spaces $\left\{x_{2}<\frac{1}{2}\right\}$ and $\left\{x_{2}>\frac{1}{2}\right\}$.

Suppose now that $\Lambda$ is a spectrum for $\Omega$. Let $\Pi$ be the set constructed from $\Lambda$ in Section 6, and $\theta(s)$ be the function on $\Pi$ given by Lemma 6.2. Let $\Lambda^{\prime}$ be the spectrum for $\Omega$ constructed from $\Lambda$ in Section 7 , and $\Lambda^{\prime \prime}$ be the spectrum constructed from $\Lambda^{\prime}$ in Section 12. We shall continue to use the notations $\Pi_{j}, \theta_{j}, \Lambda_{j}^{\prime}, \Lambda_{j}^{\prime \prime}$ and $\Lambda_{\infty}^{\prime \prime}$ with the same meaning as in the previous sections.

Our goal in the present section is to prove that, under the assumptions above, the function $\theta(s)$ is necessarily constant on $\Pi$.

14.2. It will be convenient to introduce the following notation. Let

$$
G:=(\mathbb{Z} \times \mathbb{R}) \cup(\mathbb{R} \times \mathbb{Z}),
$$

and

$$
G_{0}:=(\mathbb{Z} \times \mathbb{R}) \cup(\mathbb{R} \times(\mathbb{Z} \backslash\{0\})) .
$$

Lemma 14.1. Let $\Pi_{j}(0 \leqslant j<\infty)$ be one of the components of $\Pi$, and let $0 \leqslant k \leqslant \infty$, $k \neq j$. Then we have

$$
\Lambda_{k}^{\prime \prime} \subset \mathbb{R} \times \bigcap_{s \in \Pi_{j}}\left(s+G_{0}\right) .
$$

Also, if the set $\Lambda_{k}^{\prime \prime}$ is not empty, then we have

$$
\Pi_{j} \subset \bigcap_{(t, s) \in \Lambda_{k}^{\prime \prime}}\left(s+G_{0}\right) .
$$

In fact, each one of (14.3) and (14.4) is just a reformulation of condition (12.8). Hence Lemma 14.1 is a consequence of Lemma 12.3.

14.3.

Lemma 14.2. If for some $0 \leqslant k \leqslant \infty$, the set $\Lambda_{k}^{\prime \prime}$ is not empty, then

$$
\Lambda_{k}^{\prime \prime}-\Lambda_{k}^{\prime \prime} \not \subset \mathbb{R} \times G \text {. }
$$

Proof. The proof is very similar to that of Corollary 11.5, and therefore it will only be outlined. The proof involves several steps.

Step 1. Let $\left(t_{0}, s_{0}\right)$ be a point in $\Lambda_{k}^{\prime \prime}$, and let $f$ be the function defined by (17.4). Then the Fourier expansion

$$
f=\frac{1}{|\Omega|} \sum_{\lambda \in \Lambda^{\prime \prime}} \widehat{f}(\lambda) e_{\lambda}
$$


of $f$ with respect to the spectrum $\Lambda^{\prime \prime}$ consists only of terms corresponding to $\lambda \in \Lambda_{k}^{\prime \prime}$. This follows from Lemma 12.2 and the expression (7.5) for the Fourier transform of $f$.

Step 2. We have

$$
\Lambda_{k}^{\prime \prime}-\Lambda_{k}^{\prime \prime} \not \subset \mathbb{R} \times \mathbb{Z} \times \mathbb{R}
$$

Indeed, if this is not true then by translating $\Lambda$ we may assume that $\Lambda_{k}^{\prime \prime} \subset \mathbb{R} \times \mathbb{Z} \times \mathbb{R}$. Hence from the Fourier expansion (14.6) it follows (Lemma 2.1) that $f$ coincides a.e. on $\Omega$ with a function $\tilde{f}$ on $\mathbb{R}^{3}$ which is periodic with respect to the vector $(0,1,0)$. As in the proof of Lemma 11.2 this leads to a contradiction to the assumption that int $(\Omega)$ intersects both half-spaces $\left\{x_{2}<\frac{1}{2}\right\}$ and $\left\{x_{2}>\frac{1}{2}\right\}$.

Step 3. We have

$$
\Lambda_{k}^{\prime \prime}-\Lambda_{k}^{\prime \prime} \not \subset \mathbb{R} \times \mathbb{R} \times \mathbb{Z}
$$

In the same way, if this does not hold then by translating $\Lambda$ we can assume that $\Lambda_{k}^{\prime \prime} \subset$ $\mathbb{R} \times \mathbb{R} \times \mathbb{Z}$. As in Step 2 this implies that $f$ coincides a.e. on $\Omega$ with a function $\tilde{f}$ on $\mathbb{R}^{3}$ which is periodic with respect to the vector $(0,0,1)$. As in the proof of Lemma 11.3, this together with Lemma 10.2 implies that $\Omega$ must be a prism, a contradiction.

Step 4. We have

$$
\Lambda_{k}^{\prime \prime}-\Lambda_{k}^{\prime \prime} \not \subset \mathbb{R} \times G .
$$

This follows by combining (14.7), (14.8) and Lemma 11.4.

14.4

Lemma 14.3. Let $s, s^{\prime}, s^{\prime \prime}$ be three points in $\mathbb{R}^{2}$, and

$$
X=(s+G) \cap\left(s^{\prime}+G\right) \cap\left(s^{\prime \prime}+G\right) .
$$

If the points $s, s^{\prime}, s^{\prime \prime}$ are distinct modulo $\mathbb{Z}^{2}$, then $X-X \subset G$.

This is not difficult to verify, and we omit the details.

Lemma 14.4. Suppose that there is a component $\Pi_{j}$ of the set $\Pi(0 \leqslant j<\infty)$ such that for any $0 \leqslant k \leqslant \infty, k \neq j$, the set $\Lambda_{k}^{\prime \prime}$ is empty. Then $\Pi=\Pi_{j}$, namely $\Pi_{j}$ is the unique component of $\Pi$, and so the function $\theta(s)$ is constant on $\Pi$.

Proof. The assumption means that $\Lambda^{\prime \prime}=\Lambda_{j}^{\prime \prime}$. By (12.6) we therefore have

$$
\Lambda^{\prime \prime} \subset\left(\mathbb{Z}+\theta_{j}\right) \times \mathbb{R}^{2} .
$$

Consider the set of all points $s \in \mathbb{R}^{2}$ for which there is $t \in \mathbb{Z}+\theta_{j}$ such that $(t, s) \in \Lambda^{\prime \prime}$. We claim that this set must contain at least three points which are distinct modulo $\mathbb{Z}^{2}$. Indeed, if this is not true then the spectrum $\Lambda^{\prime \prime}$ is contained in a union of two sets of the form

$$
\left(\mathbb{Z}+\theta_{j}\right) \times\left(\mathbb{Z}^{2}+s\right), \quad s \in \mathbb{R}^{2} .
$$

But this would imply that $\Lambda^{\prime \prime}$ can be covered by the union of two translates of $\mathbb{Z}^{3}$, which is not possible according to Lemma 13.2 since $\Omega$ is not a prism (notice that $\Sigma$ contains the cube $I \times I$, so we may use Lemma 13.2). Hence there must exist three points $(t, s)$, $\left(t^{\prime}, s^{\prime}\right),\left(t^{\prime \prime}, s^{\prime \prime}\right)$ in the spectrum $\Lambda^{\prime \prime}$, such that $s, s^{\prime}, s^{\prime \prime}$ are distinct modulo $\mathbb{Z}^{2}$.

Let $\Pi_{k}, 0 \leqslant k<\infty$, be any one of the components of $\Pi$ other than $\Pi_{j}$. Then by applying (14.4) (with $j, k$ interchanged) we obtain

$$
\Pi_{k} \subset(s+G) \cap\left(s^{\prime}+G\right) \cap\left(s^{\prime \prime}+G\right) .
$$


Using Lemma 14.3 this implies that $\Pi_{k}-\Pi_{k} \subset G$, which is impossible due to Corollary 11.5. It follows that $\Pi_{j}$ must be the unique component of $\Pi$. This means that $\theta(s)=\theta_{j}$ for all $s \in \Pi$, thus $\theta(s)$ is constant on $\Pi$. The lemma is therefore proved.

14.5. At this point it will be useful to introduce the following:

Definition 14.5. Let $\left(s_{0}, s_{0}^{\prime}\right)$ be a pair of points in $\mathbb{R}^{2}$ such that $s_{0}^{\prime}-s_{0} \notin G$. If $\left(s_{1}, s_{1}^{\prime}\right)$ is another pair of points in $\mathbb{R}^{2}$, then we say that $\left(s_{1}, s_{1}^{\prime}\right)$ is dual to $\left(s_{0}, s_{0}^{\prime}\right)$ if the following conditions are satisfied:

(i) $s_{1}-s_{0}^{\prime}$ and $s_{1}^{\prime}-s_{0}$ are both in $\mathbb{Z} \times \mathbb{R}$;

(ii) $s_{1}-s_{0}$ and $s_{1}^{\prime}-s_{0}^{\prime}$ are both in $\mathbb{R} \times \mathbb{Z}$.

For example, consider the pair $\left(s_{0}, s_{0}^{\prime}\right)$ given by $s_{0}=(0,0), s_{0}^{\prime}=(\alpha, \beta)$, where $\alpha, \beta$ are two real numbers which are both not in $\mathbb{Z}$. Then the pair $\left(s_{1}, s_{1}^{\prime}\right)$ given by $s_{1}=(\alpha, 0)$, $s_{1}^{\prime}=(0, \beta)$ is dual to $\left(s_{0}, s_{0}^{\prime}\right)$.

It is not difficult to check that the duality relation just defined satisfies the following properties:

1. If $\left(s_{1}, s_{1}^{\prime}\right)$ is dual to $\left(s_{0}, s_{0}^{\prime}\right)$ then, since it was assumed that $s_{0}^{\prime}-s_{0} \notin G$, it follows that also $s_{1}^{\prime}-s_{1} \notin G$.

2. The duality relation is symmetric, that is, if $\left(s_{1}, s_{1}^{\prime}\right)$ is dual to $\left(s_{0}, s_{0}^{\prime}\right)$, then also $\left(s_{0}, s_{0}^{\prime}\right)$ is dual to $\left(s_{1}, s_{1}^{\prime}\right)$.

3 . Whether two given pairs are dual to each other or not depends only on the congruence classes of the points modulo $\mathbb{Z}^{2}$. In other words, if $\left(s_{1}, s_{1}^{\prime}\right)$ and $\left(s_{2}, s_{2}^{\prime}\right)$ are two pairs such that $s_{2}-s_{1}$ and $s_{2}^{\prime}-s_{1}^{\prime}$ are both in $\mathbb{Z}^{2}$, and if $\left(s_{1}, s_{1}^{\prime}\right)$ is dual to a certain pair $\left(s_{0}, s_{0}^{\prime}\right)$, then also $\left(s_{2}, s_{2}^{\prime}\right)$ is dual to $\left(s_{0}, s_{0}^{\prime}\right)$.

4. For every pair $\left(s_{0}, s_{0}^{\prime}\right)$ such that $s_{0}^{\prime}-s_{0} \notin G$ there exists a dual pair $\left(s_{1}, s_{1}^{\prime}\right)$, and this dual pair is unique modulo $\mathbb{Z}^{2}$.

The reason for introducing the duality relation above is the following:

Lemma 14.6. Let $\left(s_{0}, s_{0}^{\prime}\right)$ be a pair of points in $\mathbb{R}^{2}$ such that $s_{0}^{\prime}-s_{0} \notin G$. Then

$$
\left(s_{0}+G\right) \cap\left(s_{0}^{\prime}+G\right)=\mathbb{Z}^{2}+\left\{s_{1}, s_{1}^{\prime}\right\},
$$

where $\left(s_{1}, s_{1}^{\prime}\right)$ is any pair which is dual to $\left(s_{0}, s_{0}^{\prime}\right)$.

This can be checked easily. It is also easy to see that Lemma 14.6 implies:

Lemma 14.7. Let $\left(s_{0}, s_{0}^{\prime}\right)$ and $\left(s_{1}, s_{1}^{\prime}\right)$ be two pairs of points in $\mathbb{R}^{2}$, such that $s_{0}^{\prime}-s_{0}$ and $s_{1}^{\prime}-s_{1}$ are both not in $G$. If the pairs $\left(s_{0}, s_{0}^{\prime}\right)$ and $\left(s_{1}, s_{1}^{\prime}\right)$ are not dual to each other, then the set

$$
Y=\left(s_{0}+G\right) \cap\left(s_{0}^{\prime}+G\right) \cap\left(\mathbb{Z}^{2}+\left\{s_{1}, s_{1}^{\prime}\right\}\right)
$$

is contained in a translate of $\mathbb{Z}^{2}$.

14.6.

Lemma 14.8. Suppose that the set $\Pi$ can be covered by the union of two translates of $\mathbb{Z}^{2}$. Then the function $\theta(s)$ is constant on $\Pi$. 
Proof. By the assumption of the lemma there exist two points $s_{0}, s_{0}^{\prime} \in \mathbb{R}^{2}$ such that

$$
\Pi \subset \mathbb{Z}^{2}+\left\{s_{0}, s_{0}^{\prime}\right\} .
$$

Due to (12.1) we have $\Lambda^{\prime} \subset \mathbb{R} \times \Pi$, and together with (14.12) this implies that $\Lambda^{\prime}$ is contained in the set

$$
\mathbb{R} \times\left(\mathbb{Z}^{2}+\left\{s_{0}, s_{0}^{\prime}\right\}\right) .
$$

Hence all the sets in (12.3), as well as their weak limit $\Lambda^{\prime \prime}$, are also contained in (14.13).

The set $\Pi$ has at least one component $\Pi_{0}$. Since by Corollary 11.5 we have $\Pi_{0}-\Pi_{0} \not \subset$ $G$, we may assume that $s_{0}, s_{0}^{\prime}$ both belong to $\Pi_{0}$ and that $s_{0}^{\prime}-s_{0} \notin G$. Hence using (14.3) for $j=0$ we conclude that

$$
\Lambda_{k}^{\prime \prime} \subset \mathbb{R} \times\left(\left(s_{0}+G\right) \cap\left(s_{0}^{\prime}+G\right)\right)
$$

for every $1 \leqslant k \leqslant \infty$. In turn, by Lemma 14.6 this implies that $\Lambda_{k}^{\prime \prime}$ is contained in a set of the form

$$
\mathbb{R} \times\left(\mathbb{Z}^{2}+\left\{s_{1}, s_{1}^{\prime}\right\}\right),
$$

where $\left(s_{1}, s_{1}^{\prime}\right)$ is a pair which is dual to $\left(s_{0}, s_{0}^{\prime}\right)$.

We conclude that for every $1 \leqslant k \leqslant \infty$, the set $\Lambda_{k}^{\prime \prime}$ is contained in both (14.13) and (14.14), hence $\Lambda_{k}^{\prime \prime}$ must be the empty set. Now Lemma 14.4 allows us to deduce that $\Pi_{0}$ is the unique component of $\Pi$, and that $\theta(s)$ is a constant function on $\Pi$. The lemma is thus proved.

Lemma 14.9. Suppose that one of the components $\Pi_{j}$ of $\Pi$ cannot be covered by the union of two translates of $\mathbb{Z}^{2}$. Then the function $\theta(s)$ is constant on $\Pi$.

Proof. The assumption means that the component $\Pi_{j}$ contains three points $s, s^{\prime}, s^{\prime \prime}$ which are distinct modulo $\mathbb{Z}^{2}$. Hence by Lemma 14.3 the set $X$ defined by (14.9) satisfies $X-X \subset G$. By (14.3), for any $0 \leqslant k \leqslant \infty, k \neq j$, we have $\Lambda_{k}^{\prime \prime} \subset \mathbb{R} \times X$, so it follows that

$$
\Lambda_{k}^{\prime \prime}-\Lambda_{k}^{\prime \prime} \subset \mathbb{R} \times G .
$$

But according to Lemma 14.2 this is possible only if $\Lambda_{k}^{\prime \prime}$ is empty. We conclude that all the sets $\Lambda_{k}^{\prime \prime}$ such that $0 \leqslant k \leqslant \infty, k \neq j$, are empty. By Lemma 14.4 this implies that $\Pi_{j}$ is the unique component of $\Pi$, and $\theta(s)$ is constant on $\Pi$, as we had to show.

14.7 .

Lemma 14.10. Suppose that the function $\theta(s)$ is not constant on $\Pi$. Then there exist two components $\Pi_{j_{0}}$ and $\Pi_{j_{1}}\left(j_{0} \neq j_{1}\right)$ of the set $\Pi$, and there are points $s_{0}, s_{0}^{\prime} \in \Pi_{j_{0}}$ and $s_{1}, s_{1}^{\prime} \in \Pi_{j_{1}}$, such that:

(i) $\Pi_{j_{0}}$ is contained in the set

$$
X_{0}:=\mathbb{Z}^{2}+\left\{s_{0}, s_{0}^{\prime}\right\}
$$

while $\Pi_{j_{1}}$ is contained in

$$
X_{1}:=\mathbb{Z}^{2}+\left\{s_{1}, s_{1}^{\prime}\right\}
$$

(ii) $\Lambda_{j_{0}}^{\prime \prime} \subset\left(\mathbb{Z}+\theta_{j_{0}}\right) \times X_{0}$ and $\Lambda_{j_{1}}^{\prime \prime} \subset\left(\mathbb{Z}+\theta_{j_{1}}\right) \times X_{1}$;

(iii) the two pairs $\left(s_{0}, s_{0}^{\prime}\right)$ and $\left(s_{1}, s_{1}^{\prime}\right)$ are dual to each other;

(iv) $\Lambda_{k}^{\prime \prime}$ is empty for every $0 \leqslant k \leqslant \infty, k \neq j_{1}, k \neq j_{2}$. 
Proof. Assume that the function $\theta(s)$ is not constant on $\Pi$. Let $\Pi_{j_{0}}$ be one of the components of $\Pi$. By Corollary 11.5 we have $\Pi_{j_{0}}-\Pi_{j_{0}} \not \subset G$, hence there exist two points $s_{0}, s_{0}^{\prime}$ in $\Pi_{j_{0}}$ such that $s_{0}^{\prime}-s_{0} \notin G$. Observe that by Lemma 14.9 the component $\Pi_{j_{0}}$ must be contained in the union of two translates of $\mathbb{Z}^{2}$, which are necessarily given by $\mathbb{Z}^{2}+s_{0}$ and $\mathbb{Z}^{2}+s_{0}^{\prime}$. That is,

$$
\Pi_{j_{0}} \subset \mathbb{Z}^{2}+\left\{s_{0}, s_{0}^{\prime}\right\}
$$

By Lemma 14.8, the set $\Pi$ cannot be covered by the union of two translates of $\mathbb{Z}^{2}$. Hence the set $\Pi$ must contain some point $s_{1}$ which is distinct modulo $\mathbb{Z}^{2}$ from both $s_{0}$ and $s_{0}^{\prime}$. According to (14.17), the new point $s_{1}$ cannot belong to $\Pi_{j_{0}}$, hence it belongs to some other components $\Pi_{j_{1}}$.

Using (14.3) it follows that for every $0 \leqslant k \leqslant \infty, k \neq j_{0}, k \neq j_{1}$, we have

$$
\Lambda_{k}^{\prime \prime} \subset \mathbb{R} \times\left(\left(s_{0}+G\right) \cap\left(s_{0}^{\prime}+G\right) \cap\left(s_{1}+G\right)\right) .
$$

But then Lemma 14.3 implies that $\Lambda_{k}^{\prime \prime}-\Lambda_{k}^{\prime \prime} \subset \mathbb{R} \times G$. According to Lemma 14.2 this is not possible unless $\Lambda_{k}^{\prime \prime}$ is empty. We conclude that all the sets $\Lambda_{k}^{\prime \prime}$, where $0 \leqslant k \leqslant \infty$, $k \neq j_{0}, k \neq j_{1}$, are empty.

Due to Corollary 11.5, the component $\Pi_{j_{1}}$ cannot be contained in the set $\mathbb{Z}^{2}+s_{1}$, hence there is another point $s_{1}^{\prime}$ in $\Pi_{j_{1}}$ which is not congruent to $s_{1}$ modulo $\mathbb{Z}^{2}$. It then follows from Lemma 14.9 that

$$
\Pi_{j_{1}} \subset \mathbb{Z}^{2}+\left\{s_{1}, s_{1}^{\prime}\right\} .
$$

In turns, this implies that we must have $s_{1}^{\prime}-s_{1} \notin G$, again by Corollary 11.5.

Recalling the definition of the sets $\Lambda_{j_{0}}^{\prime \prime}$ and $\Lambda_{j_{1}}^{\prime \prime}$, the conditions (14.17) and (14.18) now imply that the property (ii) in the lemma is satisfied.

It remains to show that the pairs $\left(s_{0}, s_{0}^{\prime}\right)$ and $\left(s_{1}, s_{1}^{\prime}\right)$ are dual to each other. If this is not the case, then by Lemma [14.7 the set $Y$ defined by (14.11) is contained in a translate of $\mathbb{Z}^{2}$. But we have $\Lambda_{j_{1}}^{\prime \prime} \subset\left(\mathbb{Z}+\theta_{j_{1}}\right) \times Y$, due to (14.3) and property (ii). This implies that $\Lambda_{j_{1}}^{\prime \prime}-\Lambda_{j_{1}}^{\prime \prime} \subset \mathbb{Z} \times \mathbb{Z}^{2}$, and consequently $\Lambda_{j_{1}}^{\prime \prime}$ must be empty by Lemma 14.2. In a completely similar way we can also deduce that $\Lambda_{j_{0}}^{\prime \prime}$ must be empty. But this yields that all the sets $\Lambda_{k}^{\prime \prime}$, for every $0 \leqslant k \leqslant \infty$, are empty, which is impossible since $\Lambda^{\prime \prime}$ cannot be empty being a spectrum for $\Omega$. This contradiction confirms that $\left(s_{0}, s_{0}^{\prime}\right)$ and $\left(s_{1}, s_{1}^{\prime}\right)$ must be dual to each other, and concludes the proof.

14.8 .

Lemma 14.11. The function $\theta(s)$ is necessarily constant on $\Pi$.

Proof. Suppose to the contrary that this is not the case. Then by Lemma 14.10 there are two components $\Pi_{j_{0}}$ and $\Pi_{j_{1}}\left(j_{0} \neq j_{1}\right)$ of the set $\Pi$, and there are points $s_{0}, s_{0}^{\prime} \in \Pi_{j_{0}}$ and $s_{1}, s_{1}^{\prime} \in \Pi_{j_{1}}$ satisfying all the properties (i) (iv) of that lemma.

By translating the spectrum $\Lambda$ by a vector in $\{0\} \times \mathbb{R}^{2}$ we may assume that $s_{0}=(0,0)$. Since $s_{0}^{\prime}-s_{0} \notin G$, we have $s_{0}^{\prime}=(\alpha, \beta)$ for certain real numbers $\alpha, \beta$ none of which is an integer. Since the pair $\left(s_{1}, s_{1}^{\prime}\right)$ is dual to $\left(s_{0}, s_{0}^{\prime}\right)$, it follows that $s_{1}$ and $s_{1}^{\prime}$ are congruent modulo $\mathbb{Z}^{2}$ to the points $(\alpha, 0)$ and $(0, \beta)$ respectively. In other words, we have $s_{1} \in \mathbb{Z}^{2}+(\alpha, 0)$ and $s_{1}^{\prime} \in \mathbb{Z}^{2}+(0, \beta)$. 
By further translating $\Lambda$ by a vector in $\mathbb{R} \times\{(0,0)\}$ we may also assume that $\theta_{j_{0}}=0$. It will be convenient to denote $\theta:=\theta_{j_{1}}$ (notice that we then have $0<\theta<1$, since $\theta_{j_{0}}$ and $\theta_{j_{1}}$ are different numbers).

According to Lemma 13.1, the spectrum $\Lambda^{\prime \prime}$ cannot contain the whole set $\mathbb{Z} \times\{(0,0)\}$. This implies that by translating $\Lambda$ once more by some vector in $\mathbb{Z} \times\{(0,0)\}$ we may additionally assume that $\Lambda^{\prime \prime}$ does not contain the origin $(0,0,0)$.

By property (ii) from Lemma 14.10 we have

$$
\Lambda_{j_{0}}^{\prime \prime} \subset \mathbb{Z} \times\left(\mathbb{Z}^{2}+\{(0,0),(\alpha, \beta)\}\right)
$$

Hence each point in $\Lambda_{j_{0}}^{\prime \prime}$ belongs to one of two possible types:

1. Points of the form $(k, n, m)$ where $k, n, m$ are integers, not all of which are zero (that $k, n, m$ cannot all be zero follows from the assumption that $\Lambda^{\prime \prime}$ does not contain the origin);

2. Points of the form $(k, n+\alpha, m+\beta)$ where $k, n, m$ are integers.

By the same property (ii) from Lemma 14.10, we also have

$$
\Lambda_{j_{1}}^{\prime \prime} \subset(\mathbb{Z}+\theta) \times\left(\mathbb{Z}^{2}+\{(\alpha, 0),(0, \beta)\}\right) .
$$

Notice that so far, we have always used (14.3) and (14.4) with the set $G_{0}$ on the right hand side actually replaced by $G$ (which is valid since $G_{0}$ is a subset of $G$ ). However, at this point the fact that $G_{0}$, and not just $G$, appears on the right hand side of (14.3) will be important. We apply (14.3) with $j=j_{0}$ and $k=j_{1}$, and use the assumption that $(0,0)=s_{0} \in \Pi_{j_{0}}$, to conclude that

$$
\Lambda_{j_{1}}^{\prime \prime} \subset \mathbb{R} \times G_{0}
$$

It then follows from (14.20) and (14.21) that also each point in $\Lambda_{j_{1}}^{\prime \prime}$ belongs to one of two possible types:

3. Points of the form $(k+\theta, n+\alpha, m)$ where $k, n, m$ are integers, and $m$ is non-zero (that $m$ cannot be zero follows from (14.21) and the fact that $\alpha$ is not an integer);

4. Points of the form $(k+\theta, n, m+\beta)$ where $k, n, m$ are integers.

By property (iv) of Lemma 14.10, the spectrum $\Lambda^{\prime \prime}$ is the union of the two disjoint sets $\Lambda_{j_{0}}^{\prime \prime}$ and $\Lambda_{j_{1}}^{\prime \prime}$. We conclude that each point of $\Lambda^{\prime \prime}$ belongs to one of the four types 1 , 2, 3 and 4 described above.

Now consider the function

$$
f(x, y, z):=\mathbb{1}_{I}(x) \mathbb{1}_{I}(y) \mathbb{1}_{I}(z), \quad(x, y, z) \in \mathbb{R}^{3},
$$

where $I=\left[-\frac{1}{2}, \frac{1}{2}\right]$, namely, $f$ is the indicator function of the unit cube in $\mathbb{R}^{3}$. Then $f$ is supported by $\Omega$. Consider the Fourier expansion

$$
f=\frac{1}{|\Omega|} \sum_{\lambda \in \Lambda^{\prime \prime}} \widehat{f}(\lambda) e_{\lambda}
$$

of $f$ with respect to the spectrum $\Lambda^{\prime \prime}$. Since we have

$$
\widehat{f}(t, u, v)=\widehat{\mathbb{1}}_{I}(t) \widehat{\mathbb{1}}_{I}(u) \widehat{\mathbb{1}}_{I}(v), \quad(t, u, v) \in \mathbb{R}^{3},
$$

it follows that $\widehat{f}(t, u, v)=0$ whenever at least one of $t, u, v$ is a non-zero integer. This implies that $\widehat{f}$ vanishes on all the points of $\Lambda^{\prime \prime}$ which belong to types 1 and 3 . Hence 
only exponentials $e_{\lambda}$ such that $\lambda$ is of type 2 or 4 may have a non-zero coefficient in the expansion (14.22).

It follows (Lemma 2.1) that the right-hand side of (14.22) is a function $\tilde{f}$ of the form

$$
\tilde{f}(x, y, z)=e^{2 \pi i(\alpha y+\beta z)} g(x, y, z)+e^{2 \pi i(\theta x+\beta z)} h(x, y, z), \quad(x, y, z) \in \mathbb{R}^{3},
$$

where $g$ and $h$ are $\mathbb{Z}^{3}$-periodic functions, and $f$ coincides with $\tilde{f}$ a.e. on $\Omega$. Notice that it follows from (14.23) that the function $|\tilde{f}|$ is periodic with respect to the vector $(0,0,1)$. Since we have $|\tilde{f}|=|f|=1$ a.e. on $I \times I \times I$, the periodicity of $|\tilde{f}|$ implies that $|\tilde{f}|=1$ a.e. on $I \times I \times \mathbb{R}$. Hence $|f|=|\tilde{f}|=1$ a.e. on the set $\Omega \cap(I \times I \times \mathbb{R})$. On the other hand, by its definition $f$ vanishes on the set

$$
\Omega \cap(I \times I \times(\mathbb{R} \backslash I)),
$$

so the latter set must have measure zero. We conclude that

$$
\Omega \cap(I \times I \times \mathbb{R})=I \times I \times I .
$$

Since $\Omega$ contains the prism $I \times \Sigma$, and since $\left\{\frac{1}{2}\right\} \times I$ and $\left\{-\frac{1}{2}\right\} \times I$ are facets of $\Sigma$, it follows from (14.24) that $\Sigma=I \times I$. Moreover, we obtain that the intersection of $\Omega$ and the slab $\mathbb{R} \times I \times \mathbb{R}$ coincides with $I \times \Sigma$. However, by Lemma 10.2 this contradicts our assumption that $\Omega$ is not a prism. This completes the proof.

\section{Spectral CONVEX polytopes in $\mathbb{R}^{3}$ tile By tRanslations}

Based on the results obtained in the previous sections, we can now deduce:

Theorem 15.1. Let $\Omega$ be a convex polytope in $\mathbb{R}^{3}$. If $\Omega$ is spectral, then it tiles by translations.

15.1. By Theorems 3.1 and 4.1, the polytope $\Omega$ must be centrally symmetric and have centrally symmetric facets. Since Theorem 15.1 was already proved in the case when $\Omega$ is a prism (Theorem 9.1), it remains to consider the case when $\Omega$ is not a prism.

Lemma 15.2. Let $\Omega$ be a convex polytope in $\mathbb{R}^{3}$, centrally symmetric and with centrally symmetric facets, which is not a prism. If $\Lambda$ is a spectrum of $\Omega$, then

$$
\left\langle\Lambda-\Lambda, \tau_{F}\right\rangle \subset \mathbb{Z}
$$

for every facet $F$ of $\Omega$.

This result is the three-dimensional analog of Lemma 8.2. By combining Lemma 15.2 with Corollary 5.3 we immediately obtain that $\Omega$ tiles by translations, hence it only remains to prove the lemma.

15.2. Lemma 15.2 is a direct consequence of our previous results:

Proof of Lemma 15.2. Let $F$ be a facet of $\Omega$. We must show that if $\Lambda$ is a spectrum of $\Omega$, then it satisfies condition (15.1). Since $\Omega$ is not a prism, we may use Lemma 10.1 to select a subfacet $A$ of $F$ such that $\operatorname{int}(\Omega)$ intersects each one of the two open half-spaces bounded by the hyperplane $H_{F, A}$.

By applying an affine transformation we may suppose that $\Omega$ is in our "standard position", namely, $\Omega=-\Omega, F=\left\{\frac{1}{2}\right\} \times \Sigma$ where $\Sigma$ is a convex polygon in $\mathbb{R}^{2}, \Sigma=-\Sigma$, and $A=\left\{\frac{1}{2}\right\} \times\left\{\frac{1}{2}\right\} \times I$, where $I=\left[-\frac{1}{2}, \frac{1}{2}\right]$. The hyperplane $H_{F, A}$ is therefore given by 
$\left\{x_{2}=\frac{1}{2}\right\}$, and hence $\operatorname{int}(\Omega)$ intersects both half-spaces $\left\{x_{2}<\frac{1}{2}\right\}$ and $\left\{x_{2}>\frac{1}{2}\right\}$. We also have $\tau_{F}=(1,0,0)$, so that condition (15.1) becomes

$$
\Lambda-\Lambda \subset \mathbb{Z} \times \mathbb{R}^{2}
$$

Let $\Pi$ be the set constructed from $\Lambda$ in Section 6 , and $\theta(s)$ be the function on $\Pi$ given by Lemma 6.2. Since all the assumptions of Section 14 are satisfied, we may apply Lemma 14.11, which yields that the function $\theta(s)$ is constant on $\Pi$. By Corollary 7.3 this implies that (15.2) holds, which concludes the proof.

\section{UNIQUENESS OF THE SPECTRUM}

The approach that was used above to prove that in dimensions $d=2,3$ any spectral convex polytope $\Omega$ can tile by translations, also allows us to establish that, except in the case when $\Omega$ is a prism, the spectrum is unique up to translation.

16.1. To prove this we use the following lemma, which is valid in any dimension $d$ (not just $d=2,3)$.

Lemma 16.1. Let $\Omega \subset \mathbb{R}^{d}$ be a convex polytope, centrally symmetric and with centrally symmetric facets. Suppose that $\Omega$ has a spectrum $\Lambda$ satisfying (5.2) for every facet $F$ of $\Omega$. Then $\Lambda$ is a translate of the lattice $T^{*}$, the dual of the lattice $T$ given by (5.1).

Proof. By Corollary 5.3, the set $T$ given by (5.1) is a lattice, and $\Omega+T$ is a tiling. Hence by Fuglede's theorem the dual lattice $T^{*}$ is a spectrum for $\Omega$. By translating $\Lambda$ we may assume that it contains the origin. So (5.2) implies that

$$
\langle\Lambda, \tau\rangle \subset \mathbb{Z}, \quad \tau \in T .
$$

This means that $\Lambda$ is a subset of $T^{*}$. But since no proper subset of a spectrum can also be a spectrum, we must therefore have $\Lambda=T^{*}$. The lemma is thus proved.

From this lemma we immediately obtain the following sufficient condition for a spectral convex polytope to admit a unique spectrum up to translation:

Corollary 16.2. Let $\Omega \subset \mathbb{R}^{d}$ be a convex polytope, centrally symmetric and with centrally symmetric facets. Assume that $\Omega$ is spectral, and that condition (5.2) is satisfied for every spectrum $\Lambda$ of $\Omega$ and every facet $F$ of $\Omega$. Then $\Omega$ has a unique spectrum up to translation. More specifically, every spectrum $\Lambda$ of $\Omega$ is a translate of the lattice $T^{*}$.

16.2. The criterion just proved can now be applied to the following situations:

Theorem 16.3. Let $\Omega$ be a spectral convex polygon in $\mathbb{R}^{2}$ which is not a parallelogram. Then $\Omega$ admits a unique spectrum up to translation.

Theorem 16.4. Let $\Omega$ be a spectral convex polytope in $\mathbb{R}^{3}$ which is not a prism. Then $\Omega$ admits a unique spectrum up to translation.

Indeed, by Theorems 3.1 and 4.1, the polytope $\Omega$ must be centrally symmetric and have centrally symmetric facets. Hence Theorem 16.3 follows from Lemma 8.2 and Corollary 16.2, while Theorem 16.4 is a consequence of Lemma 15.2 and Corollary 16.2.

Remark that the assumptions that $\Omega$ is not a parallelogram in $\mathbb{R}^{2}$, and that it is not a prism in $\mathbb{R}^{3}$, are necessary in these results. Indeed, we have seen in Example 6.5 that if $\Omega$ is a prism, then it admits infinitely many non translation-equivalent spectra. 


\section{REMARKS AND OPEN PROBLEMS}

17.1. It would be interesting to extend Theorem 1.2 to dimensions $d \geqslant 4$.

Problem 17.1. Let $\Omega$ be a convex polytope in $\mathbb{R}^{d}(d \geqslant 4)$. Prove that if $\Omega$ is spectral, then it can tile the space by translations.

We know (Theorems 3.1 and 4.1) that such an $\Omega$ must be centrally symmetric and have centrally symmetric facets.

Using our previous results, the assertion in Problem 17.1 can be verified for the class of four-dimensional convex prisms (the polytopes $\Omega \subset \mathbb{R}^{4}$ which can be expressed as the Minkowski sum of a three-dimensional convex polytope and a line segment):

Theorem 17.2. Let $\Omega$ be a convex prism in $\mathbb{R}^{4}$. If $\Omega$ is spectral, then it can tile by translations.

Indeed, this follows from a combination of Theorems 9.2 and 15.1, in the same way as we have deduced Theorem 9.1 from Theorems 8.1 and 9.2 .

17.2. It is conceivable that Problem 17.1 could be solved in the general case by an appropriate development of our approach. However, there are certain difficulties which should be addressed in extending our proof to higher dimensions.

One problem is to identify the class of polytopes that would play the role of the parallelograms in two dimensions, and of the prisms in three dimensions. The spectral polytopes in these classes do not have a unique spectrum up to translation, and it was therefore necessary to exclude them in Lemmas 8.2 and 15.2, and, for $d=3$, to prove by a different method that they can tile by translations (Theorem 9.1).

Another problem in higher dimensions might be to obtain an analog of Lemma 12.3. In that lemma we have used the fact that in three dimensions, all the subfacets of $\Omega$ are line segments, and hence in particular they are also centrally symmetric. However, a spectral convex polytope $\Omega$ in $\mathbb{R}^{d}(d \geqslant 4)$ need not have centrally symmetric $k$ dimensional faces, for any $2 \leqslant k \leqslant d-2$ (see Section 4.1).

The latter problem disappears, though, if we impose the extra assumption that the convex polytope $\Omega$ is a zonotope. Thus we propose the following restricted version of Problem 17.1

Problem 17.3. Let $\Omega$ be a zonotope in $\mathbb{R}^{d}(d \geqslant 4)$. Prove that if $\Omega$ is spectral, then it tiles by translations.

17.3. It would also be interesting to know whether the conclusion of Theorem 1.2 is true for any convex body $\Omega$ (not assumed a priori to be a polytope). The paper [IKT03. contains a proof that, in two dimensions, a spectral convex body $\Omega$ must be a polygon. As far as we know, no such a result has been proved in dimensions $d \geqslant 3$.

Problem 17.4. Let $\Omega$ be a convex body in $\mathbb{R}^{d}$. Prove that if $\Omega$ is a spectral set, then it must be a polytope.

It is known [IKT01 that $\Omega$ cannot have a smooth boundary. Using the results in GL16] it follows that the assertion is also true if $\Omega$ is a cylindric convex body whose base has a smooth boundary. 


\section{REFERENCES}

[Ale33] A. D. Alexandrov, A theorem on convex polyhedra (Russian). Trudy Mat. Inst. Steklov 4 (1933), 87.

[Ale05] A. D. Alexandrov, Convex polyhedra. Springer-Verlag, 2005.

[Fed85] E. S. Fedorov, Elements of the study of figures (Russian). Zap. Mineral. Imper. S. Petersburgskogo Obšč. 21(2) (1885), 1-279, Izdat. Akad. Nauk SSSR, Moscow 1953.

[Fug74] B. Fuglede, Commuting self-adjoint partial differential operators and a group theoretic problem. J. Funct. Anal. 16 (1974), 101-121.

[Fug01] B. Fuglede, Orthogonal exponentials on the ball. Expo. Math. 19 (2001), no. 3, 267-272.

[GL16] R. Greenfeld, N. Lev, Spectrality and tiling by cylindric domains. J. Funct. Anal. 271 (2016), no. 10, 2808-2821.

[Gru07] P. M. Gruber, Convex and discrete geometry. Springer, 2007.

[IKP99] A. Iosevich, N. Katz, S. Pedersen, Fourier bases and a distance problem of Erdős. Math. Res. Lett. 6 (1999), no. 2, 251-255.

[IKT01] A. Iosevich, N. Katz, T. Tao, Convex bodies with a point of curvature do not have Fourier bases. Amer. J. Math. 123 (2001), no. 1, 115-120.

[IKT03] A. Iosevich, N. Katz, T. Tao, The Fuglede spectral conjecture holds for convex planar domains. Math. Res. Lett. 10 (2003), no. 5-6, 559-569.

[JP99] P. Jorgensen, S. Pedersen, Spectral pairs in Cartesian coordinates. J. Fourier Anal. Appl. 5 (1999), no. 4, 285-302.

[Kol00] M. Kolountzakis, Non-symmetric convex domains have no basis of exponentials. Illinois J. Math. 44 (2000), no. 3, 542-550.

[Kol04] M. Kolountzakis, The study of translational tiling with Fourier analysis. Fourier analysis and convexity, pp. 131-187, Birkhäuser, 2004.

[KM10] M. Kolountzakis, M. Matolcsi, Teselaciones por traslación (Spanish). Gac. R. Soc. Mat. Esp. 13 (2010), no. 4, 725-746. English version in arXiv:1009.3799.

[KP02] M. Kolountzakis, M. Papadimitrakis, A class of non-convex polytopes that admit no orthonormal basis of exponentials. Illinois J. Math. 46 (2002), no. 4, 1227-1232.

[McM70] P. McMullen, Polytopes with centrally symmetric faces. Israel J. Math. 8 (1970), 194-196.

[McM71] P. McMullen, On zonotopes. Trans. Amer. Math. Soc. 159 (1971), 91-109.

[McM80] P. McMullen, Convex bodies which tile space by translation. Mathematika 27 (1980), no. 1, 113-121.

[McM81] P. McMullen, Acknowledgement of priority: "Convex bodies which tile space by translation". Mathematika 28 (1981), no. 2, 191.

[Sch93] R. Schneider, Convex bodies: the Brunn-Minkowski theory. Cambridge University Press, 1993.

[Tao04] T. Tao, Fuglede's conjecture is false in 5 and higher dimensions. Math. Res. Lett. 11 (2004), no. $2-3,251-258$.

[Ven54] B. Venkov, On a class of Euclidean polyhedra (Russian). Vestnik Leningrad. Univ. Ser. Mat. Fiz. Him. 9 (1954), no. 2, 11-31.

[You01] R. M. Young, An introduction to nonharmonic Fourier series. Revised first edition. Academic Press, 2001.

Department of Mathematics, Bar-Ilan University, Ramat-Gan 52900, Israel

E-mail address: rachelgrinf@gmail.com

Department of Mathematics, Bar-Ilan University, Ramat-Gan 52900, Israel

E-mail address: levnir@math.biu.ac.il 